Model-theoretic imaginaries and coherent sheaves

Rajani, Ravi and Prest, Mike

2008

MIMS EPrint: 2006.410

Manchester Institute for Mathematical Sciences

School of Mathematics

The University of Manchester

\footnotetext{
Reports available from: http://eprints.maths.manchester.ac.uk/

And by contacting: The MIMS Secretary

School of Mathematics

The University of Manchester

Manchester, M13 9PL, UK
} 


\title{
Model-theoretic imaginaries and coherent sheaves
}

\author{
Ravi Rajani and Mike Prest
}

June 20, 2008

\section{Introduction}

Model theory has evolved in two sharply different directions. One is setbased, centred around pure model theory and applications to various mathematical structures: here even the language of category theory is only beginning to be heard. In contrast is the sort of model theory which is set in rather general category-theoretic, or topos-theoretic, contexts and which often looks to non-classical logics or computer science for its inspirations and applications. Our results sit in the rather sparsely populated territory between these and our hope is that this paper will help to bridge the gap between these rather different kinds of model theory. Our paper is directed mainly to setbased model-theorists in that we show how finitely presented and coherent functors arise through the imaginaries construction. This opens a door to the use of functorial techniques in model theory. Use of such techniques has proved to be enormously effective in the model theory of additive structures and we see no reason why this will not extend to the model theory of more general structures. The distances between these different sorts of model theory should not, however, be underestimated. As we ourselves found, it is quite possible to prove a result and then discover that it, or something very close to it, exists already in the literature but in a form which, without the benefit of hindsight, looks completely different.

What we do here is show the equivalence of categories of imaginaries (of various kinds) with categories of "small" (finitely generated, finitely presented, coherent) functors. We do this first for certain locally finitely presented categories and then, by localising, for much more general "definable 
categories" (categories of models of coherent theories). Then we discuss the corresponding notion of interpretation.

Some of our results may be derived from the results and proofs in [19], [17], [8] but our proofs, indeed our whole approach, is very different, being rooted in set-based model theory and the development of the model theory of additive categories. The emphases also are different: here we present the results as equivalences between categories of certain functors and certain imaginaries; in the category-based literature the final form of the results is usually a "conceptual completeness" theorem (see [16], [17], [18]) which might be expressed as an equivalence of 2-categories (see [8] for a number of examples).

Because our paper is addressed mainly to set-based model-theorists we do include proofs of category-theoretic results which can be found in (or can be deduced from) the literature. We hope that we have thereby made the paper sufficiently self-contained that it is readable to such an audience (though, inevitably, we must refer the reader elsewhere, for instance to [14] or introductory parts of [1], [3], [15], for notions that are standard for users of category theory).

Now we describe the results in more detail.

The origin of our results lies in the model theory of modules. In that context a theorem of Burke $[5,3.2 .5]$ showed how to translate between the two sorts (set-based/functor-based) of model theory. More precisely, if $R$ is a ring and Mod- $R$ is the category of right $R$-modules then there is an equivalence between, on the one hand, the category $(\bmod -R, \mathbf{A b})$ of additive functors from the category, $\bmod -R$, of finitely presented $R$-modules to that of abelian groups and, on the other hand, the category $(\operatorname{Mod}-R)^{\mathrm{eq}+}$ of ppdefined imaginaries for $R$-modules. Imaginaries belong to set-based model theory. Indeed, thinking of them as forming a category was a novel step which was taken by Herzog [6] when he defined the category $(\mathbf{M o d}-R)^{\mathrm{eq}+}$. This equivalence has the consequence that there are two quite different languages for talking about the same circle of ideas. More important, it allows an effective transfer between model-theoretic and functor-category-theoretic ideas and it led to new results as well as improved proofs of existing ones. But all this, fruitful as it was, was set within the additive context: a context more general than modules (one may replace Mod- $R$ by any locally finitely presented additive category and one may also localise), but always with the additive structure on hom-sets available for use. 
It was natural to ask whether this, in particular the key equivalence between finitely presented functors and imaginaries, could be extended to nonadditive situations. Here we show that the answer is positive.

We state his result precisely. Fix a ring $R$. Denote by $(\operatorname{Mod}-R)^{\text {eq }+}$ the category whose objects are pairs of positive primitive (pp) formulas $\varphi / \psi$ where $\psi \rightarrow \varphi$. Such a pair defines a functor $\mathbf{M o d}-R \rightarrow \mathbf{A b}$, namely that given on objects by $M \mapsto \varphi(M) / \psi(M)$. The morphisms of $(\operatorname{Mod}-R)^{\text {eq }+}$ are the pp-definable functions between such pairs. Formally, they are equivalence classes of pp-formulas $\rho: \varphi / \psi \rightarrow \varphi^{\prime} / \psi^{\prime}$, where the notation means that, on any module $M, \rho$ defines a subset of $\varphi(M) \times \varphi^{\prime}(M)$ which induces a function from $\varphi(M) / \psi(M)$ to $\varphi^{\prime}(M) / \psi^{\prime}(M)$. The equivalence relation is, of course, that of two formulas defining the same function at each module $M$. Clearly every pp-pair defines a functor from $\bmod -R$ to $\mathbf{A b}$ (indeed from all of Mod- $R$ to $\mathbf{A b}$ ), which turns out to be finitely presented, and every morphism of (Mod- $R)^{\text {eq }+}$ defines a natural transformation between the corresponding functors. The theorem is that this is an equivalence $(\mathbf{M o d}-R)^{\text {eq }+} \simeq \mathrm{fp}(\bmod -R, \mathbf{A b})$ (we use fpC to denote the full subcategory of finitely presented objects of a category $\mathbf{C}$ ).

In fact there is a relative/localised version. First note that any functor $F \in \mathrm{fp}(\bmod -R, \mathbf{A b})$ has a unique extension to a functor in $(\mathbf{M o d}-R, \mathbf{A b})$ which commutes with directed colimits (see Lemma 2,3.1). We can denote this extension $\vec{F}$ but, in practice, we use the same notation, $F$, for this extension. Suppose that $\left\{F_{\lambda}\right\}_{\lambda}$ is a family of finitely presented objects in the category ( $\bmod -R, \mathbf{A b})$. To this family one may associate the full subcategory D of Mod- $R$ consisting of those modules $M$ such that $F_{\lambda} M=0$ for every $\lambda$. By Burke's theorem each $F_{\lambda}$ is isomorphic to one of the form $\varphi_{\lambda} / \psi_{\lambda}$ for some pair of pp formulas with $\psi_{\lambda} \rightarrow \varphi_{\lambda}$. Thus $\mathbf{D}$ is exactly the class of models of the theory $T$ obtained by adding, to axioms for $R$-modules, the implications $\varphi_{\lambda} \rightarrow \psi_{\lambda}$. To the set $\left\{F_{\lambda}\right\}_{\lambda}$ of finitely presented functors one also can associate a finite type hereditary torsion theory $\tau$ on ( $\bmod -R, \mathbf{A b})$, namely that which has torsion class generated by the $F_{\lambda}$. The full subcategory of finitely presented objects in the corresponding localisation of $(\bmod -R, \mathbf{A b})$ is equivalent to the quotient of $\mathrm{fp}(\bmod -R, \mathbf{A b})$ by the Serre subcategory of $\mathrm{fp}(\bmod -R, \mathbf{A b})$ generated by the $F_{\lambda}$. The localised version of the theorem is that this quotient category of finitely presented functors is equivalent to $\mathbf{D}^{\text {eq+}}$, where the latter category has the same objects as $(\operatorname{Mod}-R)^{\text {eq }+}$ but has, for morphisms, the equivalence classes of pp formulas which define mor- 
phisms when evaluated on members of $\mathbf{D}$. Of course, if we take the family $\left\{F_{\lambda}\right\}_{\lambda}$ to consist of only the zero functor, then we obtain the original theorem.

Taking this as our starting point, we set out to prove a non-abelian version of Burke's result for modules. Being aware of, but not conversant with, the book [19] of Makkai and Reyes, it was only later that we realised the degree of relevance of their work. Our Proposition 2.13, the equivalence of the category of coherent functors with the category of "pe-imaginaries" can be extracted from [19, Chpts. 8,9] but our approach is different and more direct (and our aims are rather different from those of [19]). We also obtain characterisations of finitely generated and finitely presented functors in terms of model-theoretic imaginaries (certain quotients of pe-defined sorts).

Thus our results are strongly related to work of Makkai and coworkers, [17], [8], [18], [19]. Their "conceptual completeness" results are expressed succinctly as equivalences of certain 2-categories but the details show how to obtain various types of theories (construed as categories) from their categories of models, and vice versa. Additive analogues of these were initiated by Herzog's observation that modules are just the exact functors on the relevant category of pp-imaginaries and have been continued in, for example, [13], [21]. Our Proposition 4.4 is a generalisation of this to the non-additive setting and is, as the referee has pointed out, the finitary case of Hu's extension [8, Thm. 5.10(ii)] of Makkai's [17, 5.1]. With [17, Sec. 6] one can also derive, by a route very different from that taken in [21], the additive version. Our emphasis here is on obtaining equivalences of certain model-theoretically (in the "classical" sense) and functorially defined categories, with an aim of making it easier to use functorial techniques and ideas to prove results in "setbased" model theory. The approach we take, combining the set-based and category-based methods, has proved to be extremely effective in the model theory of modules ([22] contains many illustrations of this) and we believe that what we have done here will make it easier to use the rich and powerful technology of functor category theory in other specific model-theoretic areas of investigation.

Fix an arbitrary (finitary) first-order language $\mathcal{L}$ and let $\mathbf{C}$ denote the category of $\mathcal{L}$-structures (in fact, we begin with any locally finitely presented category). Recall that a coherent theory is one which is axiomatised by universal implications of positive existential (pe) formulas. Following the terminology used for locally finitely presented abelian categories, we say that the category of models of a coherent theory is a definable subcategory of the 
category $\mathbf{C}$. We show that such a definable subcategory gives rise to a finite type Grothendieck topology on the category of finitely presented $\mathcal{L}$-structures and the corresponding category of sheaves is a locally finitely presented topos. Actually, because we deal with covariant functors (since pe formulas define such) we deal with "cosheaves", that is covariant functors from fp C to Set, and the corresponding notion of topology. From the viewpoint of "classical" model theory this is the natural variance. That is, in this paper, by a (pre)sheaf we mean an object of (fp $\mathbf{C}$, Set), rather than the more usual meaning of an object of ((fp $\mathbf{C})^{\mathrm{op}}$, Set) so, if one prefers, a (pre-)sheaf on $(\mathrm{fp} \mathbf{C})^{\mathrm{op}}$.

The additive case suggested that we should attempt to characterise the subcategory of finitely presented sheaves as some kind of an "eq+" category. We show that the full subcategory of finitely presented sheaves is indeed equivalent to the category with objects $\varphi / \tilde{\theta}$ where $\varphi$ and $\theta$ are pe-formulas, $\theta \subseteq \varphi \times \varphi$, and $\tilde{\theta}$ denotes the equivalence relation generated by $\theta$, and with maps given by pe-formulas $\rho: \varphi / \tilde{\theta} \rightarrow \psi / \tilde{\eta}$ which define functions, as opposed to just relations, (more generally, in the localised case, when restricted to objects of a definable subcategory $\mathbf{D} \subseteq \mathbf{C}$ ). We also obtain a similar characterisation of the category of finitely generated sheaves.

Now we overlap with results of Makkai and Reyes. The category of sheaves corresponding to a coherent theory $T$ of the kind described is a well understood coherent topos called the classifying topos of $T$, so called because of a certain universal property. Rather than studying the finitely presented objects, Makkai and Reyes had considered the full subcategory of coherent objects of this topos and had obtained the following logical characterisation of this category: the coherent objects are pairs of pe-formulas $\varphi / \theta$ where $\theta \subseteq \varphi \times \varphi$ and $T \vdash(\theta$ is an equivalence relation $)$, and the maps $\varphi / \theta \rightarrow \psi / \eta$ are pe-formulas $\rho$ satisfying $T \vdash(\rho$ defines a function $\varphi / \theta \rightarrow \psi / \eta)$.

In the abelian situation coherent and finitely presented objects coincide. In the non-abelian case, there may be finitely presented objects which are not coherent. Having realised this, it was relatively easy to derive the MakkaiReyes result from our characterisation of finitely presented objects.

In summary, given a coherent theory $T$ in a first-order language $\mathcal{L}$, there is an associated category of "positive existential imaginaries" - a functorial version of Shelah's imaginaries - which can be defined in purely categorical terms as a certain category of coherent sheaves. The objects of this category are certain functors defined on the category of finitely presented models of $T$. The requirements that these functors be coherent, finitely presented or just 
finitely generated, correspond to successively more general kinds of (possibly $\bigwedge$-definable) imaginaries. We prove the results first for the case that $\operatorname{Mod}(T)$ is a locally finitely presented category (equivalently, the category of models of a finite limit theory) and then, by localising, extend to the general case. In the last part of this paper we define and investigate those interpretations between coherent theories which are given by certain types of functor between the associated "imaginaries" categories of coherent sheaves.

Throughout this paper "definable" means definable without parameters.

The work reported in this paper will form part of the doctoral thesis of the first author, who has been supported by a MATHLOGAPS Marie Curie Fellowship (MEST-CT- 2004-5040290).

\section{Finitely presented and coherent functors}

\subsection{Categorical preliminaries}

In an arbitrary category, an object is said to be finitely presented (f.p.) if its associated covariant Hom-functor commutes with directed colimits. This can be restated as follows. An object $X$ is finitely presented if whenever $\left(\left(Y_{i}\right)_{i},\left(u_{i j}: Y_{i} \rightarrow Y_{j}\right)_{i<j}\right)$ is a directed system and $Y=\underset{\lim }{\longrightarrow} Y_{i}$ is the directed colimit, any map $f: X \rightarrow Y$ factors through some object $Y_{i}$ as $f=u_{i} g$ where $u_{i}: Y_{i} \rightarrow Y$ is the canonical colimit map. Moreover, this factorisation is essentially unique in the sense that if $f=u_{i} g^{\prime}$ for some other map $g^{\prime}$ then $g$ and $g^{\prime}$ are equalised by some diagram map $u_{i j}: Y_{i} \rightarrow Y_{j}$, that is $u_{i j} g=u_{i j} g^{\prime}$.

An object is finitely generated (f.g.) if it cannot be expressed as a directed union of proper subobjects. An object $X$ is coherent if it is f.g. and whenever $Y$ is f.g. and $f: Y \rightarrow X$ is any map, then the pullback $Y \times_{X} Y$ is finitely generated.

A set of objects $\mathcal{G}$ is a generating set for a category if for any pair of maps $f, g: X \rightarrow Y$ such that $f \neq g$, there is an object $G \in \mathcal{G}$ and a map $h: G \rightarrow X$ such that $f h \neq g h$. Furthermore, $\mathcal{G}$ is a strong generating set if, in addition, whenever $X^{\prime} \rightarrow X$ is a proper subobject of $X$, there is a $G \in \mathcal{G}$ and a map $h: G \rightarrow X$ which does not factor through the subobject. A category is locally finitely presented (LFP) if it is cocomplete and has a strong generating set of f.p. objects. Equivalently, a category is LFP if it is cocomplete and has a set of f.p. objects such that every object is a directed colimit of these (see [1, p. 17]). The following proposition gives a characterisation of f.g. and f.p. objects in 
an LFP topos, mirroring the abelian situation. A (Grothendieck) topos is simply the category of (set-valued) sheaves on a Grothendieck site, that is, on a small category with a Grothendieck topology. All these notions will be defined later. The topologies that will arise in this paper will all be of finite type, a fact which ensures that the corresponding categories of sheaves are LFP topoi. The proof of the following proposition does not mention sheaves, but uses instead certain completeness and exactness properties of topoi. In fact, a topos can be characterised as a category with certain completeness and exactness properties (this is the content of Giraud's theorem). All this is explained in the book of MacLane and Moerdijk [15].

The next results can be deduced from results in the category-theoretic literature but we include direct proofs here to make the paper more accessible to readers from set-based model theory. The first part of the result below follows from $[1,1.69]$.

Proposition 2.1. Let $\mathcal{E}$ be an LFP topos (so in particular $\mathcal{E}$ is Grothendieck) with a generating set $\mathcal{G}$ of f.p. objects.

(a) An object $X \in \mathcal{E}$ is finitely generated if and only if there are objects $G_{1}, \ldots, G_{n} \in \mathcal{G}$ and an epimorphism

$$
\coprod_{i=1}^{n} G_{i} \longrightarrow X
$$

(b) An object $X \in \mathcal{E}$ is finitely presented if and only if there are objects $G_{1}, \ldots, G_{n}, H_{1}, \ldots, H_{m} \in \mathcal{G}$ and a coequaliser diagram of the form

$$
\coprod_{i=1}^{n} G_{i} \longrightarrow \coprod_{i=1}^{m} H_{i} \longrightarrow X
$$

Proof. (a)

$(\Rightarrow)$ Write $\mathcal{G}=\left\{G_{i}\right\}_{i}$, and put $A_{i}=\operatorname{Hom}\left(G_{i}, X\right)$. Define the map

$$
\coprod_{i} G_{i}^{\left(A_{i}\right)} \stackrel{p}{\longrightarrow} X
$$

by $p_{i, f}=f$ (for $f \in A_{i}$ ). Then $p$ is epic. To see this, suppose we have two maps $g \neq h: X \rightarrow Y$. Since $\mathcal{G}$ generates the category, there is an $i$ and a map $t: G_{i} \rightarrow X$ with $g t \neq h t$. This gives $g p_{i, t} \neq h p_{i, t}$ and so $(g p)_{i, t} \neq(h p)_{i, t}$ which implies that $g p \neq h p$. 
The components of $p$ determine maps from any finite subcoproduct of $\coprod_{i} G_{i}^{\left(A_{i}\right)}$ to $X$. The images of these maps determine a directed system of subobjects of $X$, with colimit $X$. Since $X$ is f.g., $X=\operatorname{im}\left(\coprod_{i=1}^{n} G_{i} \rightarrow X\right)$ for some $G_{1}, \ldots, G_{n} \in \mathcal{G}$. So there is an epimorphism

$$
\coprod_{i=1}^{n} G_{i} \longrightarrow X
$$

as required.

$(\Leftarrow)$ Now suppose there is an epimorphism $\coprod_{i=1}^{n} G_{i} \rightarrow X$. Since a finite colimit of f.p. objects is f.p. (see [1, p. 12]), $X$, as a quotient of a f.p. object, is f.g. For if $X=\lim X_{i}$ as a directed union of subobjects, then the epimorphism factors through some subobject $X_{j}$ as $f_{i} g$ so the inclusion $f_{i}$ must be epic hence, as we're in a topos, is $\operatorname{id}_{X}$.

(b) $(\Rightarrow)$ Suppose that $X$ is finitely presented. Then in particular, $X$ is f.g. and so by (a) there must be an epimorphism $p: \coprod_{i=1}^{m} H_{i} \rightarrow X$ where $H_{i} \in \mathcal{G}$. Let

$$
Y \underset{\pi_{2}}{\stackrel{\pi_{1}}{\rightleftarrows}} \coprod_{i=1}^{m} H_{i}
$$

be the kernel pair of $p$. So

$$
Y \underset{\pi_{2}}{\stackrel{\pi_{1}}{\longrightarrow}} \coprod_{i=1}^{m} H_{i} \stackrel{p}{\longrightarrow} X
$$

is a coequaliser. Let $\left\{M_{i}\right\}_{i}$ be a directed system of subobjects of $Y$ as in (a). So each $M_{i}$ is the image of map from a finite coproduct of objects from $\mathcal{G}$ into $Y$. Let $X_{i}$ be the coequaliser of the maps

$$
M_{i} \longrightarrow Y \underset{\pi_{2}}{\stackrel{\pi_{1}}{\longrightarrow}} \coprod_{i=1}^{m} H_{i}
$$

Note that an inclusion $M_{i} \subseteq M_{j}$ induces an epimorphism $u_{i j}: X_{i} \rightarrow X_{j}$. The $X_{i}$ with the $u_{i j}$ form a directed system with $X=\underline{\lim } X_{i}$ and with the cone maps $u_{i}: X_{i} \rightarrow X$ given by the universal property of the coequaliser. Since $X$ is f.p., $\mathrm{id}_{X}$ factors through some $X_{j}$. So $\operatorname{id}_{X}=u_{j} f$ for some $f: X \rightarrow X_{j}$. There is an epimorphism

$$
\coprod_{i=1}^{l} G_{i}^{\prime} \longrightarrow M_{j}
$$


where $G_{i}^{\prime} \in \mathcal{G}$ hence, since the $G_{i}^{\prime}$ are finitely presented, there is a coequaliser diagram of the form

$$
\coprod_{i=1}^{l} G_{i}^{\prime} \longrightarrow \coprod_{i=1}^{m} H_{i} \longrightarrow X_{j}
$$

Since $X_{j}$ is a finite colimit of f.p. objects, $X_{j}$ is itself f.p. Hence the factorisation

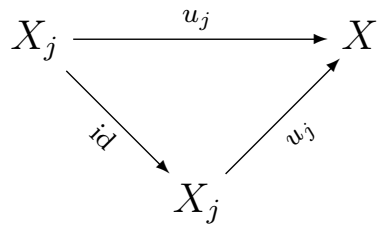

of $u_{i}$ as $u_{j}$ id is essentially unique (in the sense defined earlier). So, since also $u_{j}=u_{j}\left(f u_{j}\right)$, there is a $k \geq j$ such that $u_{j k}=u_{j k} f u_{j}$. We claim that $u_{k}: X_{k} \rightarrow X$ is an isomorphism. First note that $u_{k}\left(u_{j k} f\right)=\left(u_{k} u_{j k}\right) f=$ $u_{j} f=\operatorname{id}_{X}$. But also $\left(\left(u_{j k} f\right) u_{k}\right) u_{j k}=\left(u_{j k} f\right)\left(u_{k} u_{j k}\right)=u_{j k} f u_{j}=u_{j k}$. So since $u_{j k}$ is epic, $\left(u_{j k} f\right) u_{k}=\operatorname{id}_{X_{k}}$. So indeed $u_{k}$ is invertible. Hence $X \cong X_{k}$ and there is a coequaliser diagram

$$
\coprod_{i=1}^{n} G_{i} \longrightarrow \coprod_{i=1}^{m} H_{i} \longrightarrow X
$$

as required.

$(\Leftarrow)$ This direction is direct from the fact that a finite colimit of f.p. objects is f.p.

Corollary 2.2. Let $\mathcal{E}$ and $\mathcal{G}$ be as above. If $X$ is a coherent object of $\mathcal{E}$, then $X$ is finitely presented.

Proof. Since $X$ is f.g., there is an epimorphism $\coprod_{i=1}^{n} G_{i} \longrightarrow X$ where $G_{i} \in \mathcal{G}$. Let $G=\amalg_{i} G_{i}$ and put $Y=G \times_{X} G$. $Y$ is f.g. since $X$ is coherent and so there is an epimorphism $\coprod_{j=1}^{m} H_{j} \longrightarrow Y$. This gives us a coequaliser

$$
\coprod_{j} H_{j} \longrightarrow G \longrightarrow X
$$

Hence $X$ is f.p. as required.

A functor category is an LFP topos and is generated by the set of representable functors. So we get the following useful corollary. 
Corollary 2.3. Let $\mathbf{C}$ be a small category and let $F$ be a set-valued functor on $\mathbf{C}$.

(a) $F$ is finitely generated if and only if there are objects $C_{1}, \ldots, C_{n} \in \mathbf{C}$ and an epimorphism

$$
\coprod_{i=1}^{n} \operatorname{Hom}\left(C_{i},-\right) \longrightarrow F
$$

(b) $F$ is finitely presented if and only if there are objects $C_{1}, \ldots, C_{n}, D_{1}, \ldots, D_{m} \in$ $\mathbf{C}$ and a coequaliser diagram

$$
\coprod_{i=1}^{n} \operatorname{Hom}\left(C_{i},-\right) \Longrightarrow \coprod_{i=1}^{m} \operatorname{Hom}\left(D_{i},-\right) \longrightarrow F
$$

\subsection{Finitely generated and finitely presented functors}

We begin with a locally finitely presented category C. For instance, the category of modules over a ring, the category of groups, or of rings, et cetera. In particular, if $\mathcal{L}$ is a (finitary, but possibly many-sorted) first-order language then the category of $\mathcal{L}$-structures is such a category, as is the category of models of any finite limit theory in $\mathcal{L}$ (meaning a theory axiomatised by sentences of the form $\forall \boldsymbol{x}(\varphi(\boldsymbol{x}) \rightarrow \exists ! \boldsymbol{y} \psi(\boldsymbol{x}, \boldsymbol{y}))$ where $\varphi, \psi$ are $\Lambda$-atomic). Indeed (see $[1,5.9]$ ), conversely, every locally finitely presented category can be regarded as the category of models for a finite limit theory in a suitable language $\mathcal{L}$. Choose and fix such $\mathcal{L}$.

It may be that the category of definable sets in objects of $\mathbf{C}$ already has disjoint unions in the sense that if $U, V$ are definable sets then there is a definable set $W$ (in some sort) and definable injections $f: U \rightarrow W$, $g: V \rightarrow W$ such that $W$ is the disjoint union of the images of $f$ and $g$. If not, then we use the following device. Expand the language, to $\mathcal{L}^{\prime}$, by adding one new sort and two constants of this new sort $\left\{c_{0}, c_{1}\right\}$. Add the axiom $c_{0} \neq c_{1}$ and the sentence expressing that there are exactly two elements of the new sort. Variables of the new sort will be denoted by $x_{1}^{\prime}, x_{2}^{\prime}, \ldots$ etc. This will ensure that we can form definable disjoint unions of (finitely many) definable sets. Let $\mathbf{C}^{\prime}$ be the category (with the usual homomorphisms) of $\mathcal{L}^{\prime}$ structures satisfying these sentences and axioms for $\mathbf{C}$ (or just the category $\mathbf{C}$ if we did not add the extra sort). Then $\mathbf{C}^{\prime}$ is an LFP category with a skeletally small class fp $\mathbf{C}^{\prime}$ of finitely presented objects (see [1, p. 201]) and these have the form $M \cup\left\{c_{0}, c_{1}\right\}$ where $M$ is a f.p. object of $\mathbf{C}$. By, e.g. [1, p. 201], an object of $\mathbf{C}^{\prime}$ is f.p. if it is isomorphic to a quotient of the term 
algebra on finitely many variables by finitely many term equations and is such that the interpretations of the relation symbols have just finitely many edges. From now on we write $\mathcal{L}$ for $\mathcal{L}^{\prime}, \mathbf{C}$ for $\mathbf{C}^{\prime}$ and assume that the language was added to as above if this was necessary.

A crucial observation in what follows is that a positive existential formula, being preserved by homomorphisms, defines a functor $\mathbf{C} \rightarrow$ Set by associating to each object $C \in \mathbf{C}$ the set it defines and with the obvious action on morphisms. In particular each positive existential formula $\varphi$ is identified with an element of the functor category ( $\mathrm{fp} \mathbf{C}$, Set) (we use the same notation for the formula and the functor).

It is worth singling out the following two properties of the category $\mathbf{C}$. We say that a tuple $\boldsymbol{a}$ of elements of $A \in \mathbf{C}$ generates $A$ if for every $b \in A$ there is a term $t(\boldsymbol{x})$ of $\mathcal{L}$ such that $b=t(\boldsymbol{a})$. Proofs of these properties are easily extracted from $[1$, Sec. $5 \mathrm{~A}]$.

$\mathbf{F R}$ If $\varphi(\boldsymbol{x})$ is a finite conjunction of atomic formulas, then there is an object $A \in \operatorname{fp}(\mathbf{C})$ and a tuple of generators $\boldsymbol{a} \in \varphi(A)$ for $A$ with the property that if $B$ is any object and $\boldsymbol{b} \in \varphi(B)$ then the map $\boldsymbol{a} \mapsto \boldsymbol{b}$ extends to a morphism $A \rightarrow B$. Following the terminology used (for a more general notion) in the additive case ([23, Sec. 8.3$])$, we say that the pair $(A, \boldsymbol{a})$ is a free realisation of $\varphi$.

PF If $C \in \operatorname{fp}(\mathbf{C})$ and $\boldsymbol{c}$ is a tuple of generators for $C$, then there is a finite conjunction of atomic formulas $\varphi(\boldsymbol{x})$ with $\boldsymbol{c} \in \varphi(C)$ such that whenever $D \in \mathbf{C}$ and $\boldsymbol{d} \in \varphi(D)$, the map $\boldsymbol{c} \mapsto \boldsymbol{d}$ extends to a morphism $C \rightarrow D$. Such a formula is called a presentation formula for $C$.

Note that FR implies that any finite conjunction of atomic formulas defines a representable functor. Conversely, PF implies that any representable functor is isomorphic to a functor defined by a finite conjunction of atomic formulas.

Let $\varphi$ be a pe-formula and $R$ a subfunctor of $\varphi \times \varphi$ in (fp C, Set). Suppose $C \in \mathrm{fp} \mathbf{C}$ and $\boldsymbol{a}, \boldsymbol{b}$ are tuples from $\varphi(C)$. Then we write $R(\boldsymbol{a}, \boldsymbol{b})$ when $(\boldsymbol{a}, \boldsymbol{b}) \in R(C)$. If for every $C \in \mathrm{fp} \mathbf{C}$ it is the case that $R(C)$ is an equivalence relation on $\varphi(C)$, then we say that $R$ is a functorial equivalence relation on $\varphi$. We write $\varphi / R$ for the functor which associates to $C \in \mathrm{fp} \mathbf{C}$ the set of equivalence classes of $\varphi(C)$ modulo $R(C)$. 
Proposition 2.4. Let $F$ be a functor in (fp C, Set).

(a) Suppose that $F$ is finitely generated Then there is a positive quantifierfree $\mathcal{L}$-formula $\varphi(\boldsymbol{x})$ and a functorial equivalence relation $E(\boldsymbol{x}, \boldsymbol{y})$ such that

$$
F \cong \frac{\varphi}{E}
$$

(b) Suppose that $F$ is finitely presented Then there is a positive quantifierfree $\mathcal{L}$-formula $\varphi(\boldsymbol{x})$ and a positive existential formula $\theta(\boldsymbol{x}, \boldsymbol{y})$ such that

$$
F \cong \frac{\varphi}{\tilde{\theta}}
$$

where $\tilde{\theta}$ denotes the equivalence relation generated by $\theta$.

Proof. (a) Suppose $F$ is finitely generated. By Corollary 2.3, there are $D_{1}, \ldots, D_{m} \in \mathrm{fp} \mathbf{C}$ and an epimorphism

$$
\coprod_{j} \operatorname{Hom}\left(D_{j},-\right) \longrightarrow F
$$

Let $\varphi_{j}$ be a presentation formula for $D_{j}$ and assume without loss of generality that the $\varphi_{j}$ all have the same number of free variables. For if the largest number of free variables among the $\varphi_{j}$ is $l$ and $\varphi_{j}=\varphi_{j}\left(x_{1}, \ldots, x_{r}\right)$ where $r<l$, then simply add to $\varphi_{j}$ a conjunction of equations $x_{r+1}=x_{1} \wedge \cdots \wedge x_{l}=$ $x_{1}$.

Now let $\varphi_{j}^{\prime}\left(x_{1}, \ldots, x_{l}, x_{1}^{\prime}, \ldots, x_{m}^{\prime}\right)$ be the formula

$$
\varphi_{j}\left(x_{1}, \ldots, x_{l}\right) \wedge x_{1}^{\prime}=c_{0} \wedge \cdots \wedge x_{j}^{\prime}=c_{1} \wedge \cdots \wedge x_{m}^{\prime}=c_{0}
$$

so that $\varphi=\varphi_{1}^{\prime} \vee \cdots \vee \varphi_{m}^{\prime}$ defines the disjoint union of the sets defined by $\varphi_{1}, \ldots, \varphi_{m}$. In particular, $\varphi \cong \coprod_{j=1}^{m} \varphi_{j}$ as a coproduct of functors (note that, in the functor category, coproduct is given pointwise by disjoint union) and we have an epimorphism

$$
\varphi \stackrel{p}{\longrightarrow} F
$$

Let $R \subseteq \varphi \times \varphi$ be the functor given by

$$
M \longmapsto\left\{(\boldsymbol{x}, \boldsymbol{y}) \in \varphi(M)^{2}: p_{M}(\boldsymbol{x})=p_{M}(\boldsymbol{y})\right\}
$$

and let $E(\boldsymbol{x}, \boldsymbol{y})$ be the equivalence relation generated by $R$. Then $F \cong \varphi / E$ as required. 
(b) Now suppose that $F$ is f.p., so there is a presentation for $F$ of the form

$$
\coprod_{i=1}^{n} \operatorname{Hom}\left(C_{i},-\right) \stackrel{p}{\underset{q}{\longrightarrow}} \coprod_{j=1}^{m} \operatorname{Hom}\left(D_{j},-\right) \longrightarrow F
$$

We can write this as

$$
\coprod_{i=1}^{n} \psi_{i} \underset{q}{\stackrel{p}{\longrightarrow}} \varphi \longrightarrow F
$$

where $\varphi \cong \coprod_{j} \varphi_{j}$ as in part (a) and $\psi_{i}$ is a presentation formula for $C_{i}$.

Since $p$ and $q$ are natural transformations, their components $p_{i}$ and $q_{i}$ are determined by their actions at $C_{i}$ on the identity map $\mathrm{id}_{C_{i}}$. So if $k$ is such that $\left(p_{i}\right)_{C_{i}}\left(\operatorname{id}_{C_{i}}\right) \in \operatorname{Hom}\left(D_{k}, C_{i}\right) \simeq \varphi_{k}\left(C_{i}\right)$ then $p_{i}$ is a natural transformation $\psi_{i} \rightarrow \varphi_{k}$. So since $p_{i}$ is a natural transformation between two representable functors, it must be induced by a map $f: D_{k} \rightarrow C_{i}$. In other words $p_{i}=f^{*}$ (the latter is the notation we will use for $\operatorname{Hom}(f,-))$.

Let $\boldsymbol{c}$ be a tuple of generators for $C_{i}$ and $\boldsymbol{d}$ a tuple of generators for $D_{k}$. Let $E \in \mathrm{fp}(\mathbf{C})$ and consider the component

$$
\psi_{i}(E) \stackrel{p_{i}}{\longrightarrow} \varphi_{k}(E)
$$

For each $d_{j}$ from the tuple $\boldsymbol{d}$, there is a term $t_{j}$ such that $f\left(d_{j}\right)=t_{j}(\boldsymbol{c})$. Let $\boldsymbol{t}(\boldsymbol{c})$ be the tuple whose $j$ th component is $t_{j}(\boldsymbol{c})$. Then the action of $\left(p_{i}\right)_{E}$ on a tuple $\boldsymbol{e} \in \psi_{i}(E)$ is given simply by

$$
e \longmapsto t(e)
$$

So we see that $p_{i}$ is a definable map (that is, its graph is, uniformly over $\mathbf{C}$, a definable subset of the product of its domain and codomain). Clearly $p_{i}$ can be regarded as a definable map into $\varphi_{k}^{\prime}$ by extending the tuple $\boldsymbol{t}(\boldsymbol{x})$ in the obvious way.

Let $\theta(\boldsymbol{x}, \boldsymbol{y})$ be the relation on $\varphi$ which holds when there is an element $\boldsymbol{a} \in \coprod_{i=1}^{n} \psi_{i}$ such that $p(\boldsymbol{a})=\boldsymbol{x}$ and $q(\boldsymbol{a})=\boldsymbol{y}$. This is expressible by the formula

$$
\exists \boldsymbol{z}\left(\bigvee_{i=1}^{n}\left(\psi_{i}(\boldsymbol{z}) \wedge p_{i}(\boldsymbol{z})=\boldsymbol{x} \wedge q_{i}(\boldsymbol{z})=\boldsymbol{y}\right)\right)
$$

It should be clear that

$$
F \cong \frac{\varphi}{\tilde{\theta}}
$$

as required. 
In the additive case the corresponding results, see [5, 3.2.5], [23, Chpt. 12], require only positive primitive (pp) formulas. These are the formulas built up from atomic formulas using just conjunction and existential quantification. To obtain a converse to the above proposition we will need the following lemmas.

Lemma 2.5. Any positive primitive L-formula defines a finitely presented functor.

Proof. Let $\varphi(\boldsymbol{x})$ be the positive primitive formula $\exists \boldsymbol{y} \theta(\boldsymbol{x}, \boldsymbol{y})$ where $\theta$ is a conjunction of atomic formulas. Let $(C, \boldsymbol{c}, \boldsymbol{d})$ be a free realisation of $\theta$. Clearly $C$ has the property that if $E$ is any other object with $\boldsymbol{e} \in \varphi(E)$, there is a map $f: C \rightarrow E$ with $f(\boldsymbol{c})=\boldsymbol{e}$. So the map

$$
\operatorname{Hom}(C,-) \longrightarrow \varphi
$$

defined by

$$
f \longmapsto f(\boldsymbol{c})
$$

is epic. Hence $\varphi$ is finitely generated.

Now let $\psi\left(\boldsymbol{x}, \boldsymbol{y}_{1}, \boldsymbol{y}_{2}\right)$ be the formula $\theta\left(\boldsymbol{x}, \boldsymbol{y}_{1}\right) \wedge \theta\left(\boldsymbol{x}, \boldsymbol{y}_{2}\right)$. As a finite conjunction of atomic formulas, $\psi$ is representable, hence finitely presented. Define the map $p_{i}: \psi \rightarrow \operatorname{Hom}(C,-)$ to be that whose component at $E$ takes $\left(\boldsymbol{a}, \boldsymbol{b}_{1}, \boldsymbol{b}_{2}\right) \in \psi(E)$ to the map defined by $(\boldsymbol{c}, \boldsymbol{d}) \mapsto\left(\boldsymbol{a}, \boldsymbol{b}_{i}\right)$. Then the diagram

$$
\psi \underset{p_{2}}{\stackrel{p_{1}}{\longrightarrow}} \operatorname{Hom}(C,-) \longrightarrow \varphi
$$

is a coequaliser. Since $\psi$ and $\operatorname{Hom}(C,-)$ are f.p., $\varphi$ is also finitely presented.

Lemma 2.6. Any positive existential formula defines a finitely presented functor.

Proof. Any positive existential formula $\varphi$ is a disjunction of positive primitive formulas. We proceed by induction on the number $n$ of disjuncts. If $n=1$ then $\varphi$ is positive primitive and so finitely presented by the above. Now suppose $n>1$. Write $\varphi=\bigvee_{i=1}^{n} \varphi_{i}$ where each $\varphi_{i}$ is positive primitive. The following diagram, with the obvious maps from the conjunction to the union, is a coequaliser.

$$
\left(\bigvee_{i=1}^{n-1} \varphi_{i}\right) \wedge \varphi_{n} \longrightarrow\left(x^{\prime}=c_{0} \wedge \bigvee_{i=1}^{n-1} \varphi_{i}\right) \vee\left(x^{\prime}=c_{1} \wedge \varphi_{n}\right) \longrightarrow \varphi
$$


Both $\bigvee_{i=1}^{n-1} \varphi_{i}$ and $\left(\bigvee_{i=1}^{n-1} \varphi_{i}\right) \wedge \varphi_{n}$ have $n-1$ disjuncts so the induction hypothesis applies. It now easily follows that $\varphi$ is finitely presented since the functor in the middle is a coproduct of f.p. functors.

\section{Proposition 2.7.}

(a) Any functor of the form $\varphi / E$ where $\varphi$ is positive existential and $E$ is a functorial equivalence relation on $\varphi$ is finitely generated.

(b) Any functor of the form $\varphi / \tilde{\theta}$ where $\varphi$ is a positive existential formula and $\theta$ is a positive existential relation on $\varphi$ is finitely presented.

Proof. (a) There is a natural epimorphism $\varphi \longrightarrow \varphi / E$. Hence $\varphi / E$ is f.g. (b) Consider the diagram

$$
\theta \longrightarrow \varphi \longrightarrow \varphi / \tilde{\theta}
$$

where the maps are the obvious ones. This is clearly a coequaliser. So $\varphi / \tilde{\theta}$ is finitely presented as required.

Combining Propositions 2.4 and 2.7 we get the following corollary.

Corollary 2.8. Let $F$ be a functor in (fp C, Set).

(a) $F$ is finitely generated if and only if $F \cong \varphi / E$ where $\varphi$ is positive existential and $E$ is a functorial equivalence relation.

(b) $F$ is finitely presented if and only if it is of the form $\varphi / \tilde{\theta}$ where $\varphi$ and $\theta$ are positive existential.

We will now characterise the natural transformations between f.g. functors in terms of positive existential formulas. Let $\varphi / E_{1}$ and $\psi / E_{2}$ be two f.g. functors. A positive existential formula $\rho(\boldsymbol{x}, \boldsymbol{y})$ clearly defines a natural transformation $\varphi / E_{1} \rightarrow \psi / E_{2}$ if the following are satisfied for each $A \in$ fp C.

- For every $\boldsymbol{a} \in \varphi(A)$ there is a $\boldsymbol{b} \in \psi(A)$ with $\rho(\boldsymbol{a}, \boldsymbol{b})$.

- If $\boldsymbol{a}, \boldsymbol{a}^{\prime} \in \varphi(A)$ are such that $E_{1}\left(\boldsymbol{a}, \boldsymbol{a}^{\prime}\right)$ and for some tuples $\boldsymbol{b}, \boldsymbol{b}^{\prime}$ we have $\rho(\boldsymbol{a}, \boldsymbol{b})$ and $\rho\left(\boldsymbol{a}^{\prime}, \boldsymbol{b}^{\prime}\right)$, then $\boldsymbol{b}, \boldsymbol{b}^{\prime} \in \psi(A)$ and $E_{2}\left(\boldsymbol{b}, \boldsymbol{b}^{\prime}\right)$.

As $\rho$ is a natural transformation, $\rho_{A}$ takes an equivalence class $[\boldsymbol{a}]_{E_{1}}$ to the equivalence class $[\boldsymbol{b}]_{E_{2}}$ where $\rho(\boldsymbol{a}, \boldsymbol{b})$ holds.

Proposition 2.9. Every natural transformation in $\mathrm{fg}(\mathrm{fp} \mathbf{C}$, Set), the full subcategory of finitely generated functors, is defined by a positive existential formula. 
Proof. Let $\alpha: \varphi / E_{1} \rightarrow \psi / E_{2}$ be a natural transformation in $\mathrm{fg}(\mathrm{fp} \mathbf{C}$, Set). The positive existential formula $\varphi$ will be of the form $\bigvee_{i} \exists \boldsymbol{z} \sigma_{i}(\boldsymbol{x}, \boldsymbol{z})$ where $\sigma_{i}$ is a conjunction of atomic formulas. Let $\left(A_{i}, \boldsymbol{a}_{i}, \boldsymbol{b}_{i}\right)$ be a free realisation of $\sigma_{i}$. For each $i$ choose a representative $\boldsymbol{a}_{i}^{\prime}$ for the $E_{2}$-equivalence class $\alpha_{A_{i}}\left[\boldsymbol{a}_{i}\right]_{E_{1}}$. Since $\left(\boldsymbol{a}_{i}, \boldsymbol{b}_{i}\right)$ generates $A_{i}$, there will be a tuple $\boldsymbol{t}_{i}$ of terms such that $\boldsymbol{t}_{i}\left(\boldsymbol{a}_{i}, \boldsymbol{b}_{i}\right)=\boldsymbol{a}_{i}^{\prime}$. Let $\rho(\boldsymbol{x}, \boldsymbol{y})$ be the formula

$$
\bigvee_{i} \exists \boldsymbol{z}\left(\sigma_{i}(\boldsymbol{x}, \boldsymbol{z}) \wedge \boldsymbol{t}_{i}(\boldsymbol{x}, \boldsymbol{z})=\boldsymbol{y}\right)
$$

Suppose that $\boldsymbol{c}, \boldsymbol{c}^{\prime}$ are tuples from an object $C$ and that $\rho\left(\boldsymbol{c}, \boldsymbol{c}^{\prime}\right)$ holds. Then there is an $i$ and a tuple $\boldsymbol{d}$ such that $\sigma_{i}(\boldsymbol{c}, \boldsymbol{d})$ and $\boldsymbol{t}_{i}(\boldsymbol{c}, \boldsymbol{d})=\boldsymbol{c}^{\prime}$. So there is a map $f: A_{i} \rightarrow C$ defined by $\left(\boldsymbol{a}_{i}, \boldsymbol{b}_{i}\right) \mapsto(\boldsymbol{c}, \boldsymbol{d})$. Since $\alpha$ is a natural transformation, the following diagram commutes, where $f_{*}$ denotes the obvious induced map.
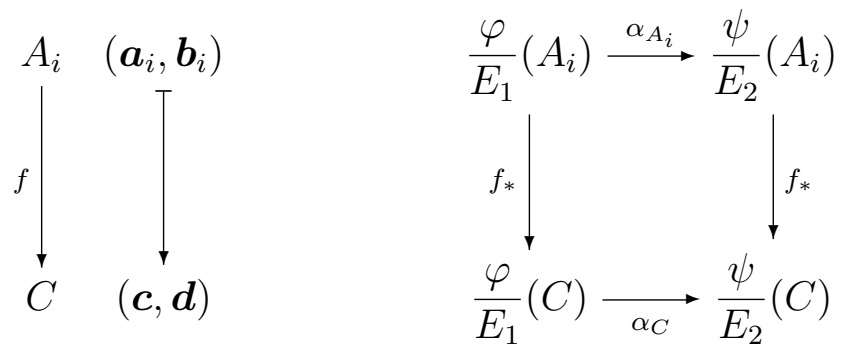

Clearly $\boldsymbol{c} \in \varphi(C)$ so we can investigate the action of $\alpha_{C}$ on the equivalence class $[\boldsymbol{c}]_{E_{1}}$.

$$
\begin{aligned}
\alpha_{C}[\boldsymbol{c}]_{E_{1}} & =\alpha_{C}\left[f\left(\boldsymbol{a}_{i}\right)\right]_{E_{1}} \\
& =\alpha_{C} f_{*}\left[\boldsymbol{a}_{i}\right]_{E_{1}} \\
& =f_{*} \alpha_{A_{i}}\left[\boldsymbol{a}_{i}\right]_{E_{1}} \\
& =f_{*}\left[\boldsymbol{a}_{i}^{\prime}\right]_{E_{2}} \\
& =f_{*}\left[\boldsymbol{t}_{i}\left(\boldsymbol{a}_{i}, \boldsymbol{b}_{i}\right)\right]_{E_{2}} \\
& =\left[f\left(\boldsymbol{t}_{i}\left(\boldsymbol{a}_{i}, \boldsymbol{b}_{i}\right)\right)\right]_{E_{2}} \\
& =\left[\boldsymbol{t}_{i}\left(f\left(\boldsymbol{a}_{i}, \boldsymbol{b}_{i}\right)\right)\right]_{E_{2}} \\
& =\left[\boldsymbol{t}_{i}(\boldsymbol{c}, \boldsymbol{d})\right]_{E_{2}} \\
& =\left[\boldsymbol{c}^{\prime}\right]_{E_{2}}
\end{aligned}
$$

So we see that if $\rho\left(\boldsymbol{c}, \boldsymbol{c}^{\prime}\right)$ holds in $C$, then $\alpha_{C}[\boldsymbol{c}]_{E_{1}}=\left[\boldsymbol{c}^{\prime}\right]_{E_{2}}$. We now check that $\rho$ defines the natural transformation $\alpha$. 
Let $\boldsymbol{a} \in \varphi(A)$. Then there is an $i$ and a tuple $\boldsymbol{b}$ such that $\sigma_{i}(\boldsymbol{a}, \boldsymbol{b})$. Clearly $\rho\left(\boldsymbol{a}, \boldsymbol{t}_{i}(\boldsymbol{a}, \boldsymbol{b})\right)$ holds. By above, this implies that $\alpha_{A}[\boldsymbol{a}]_{E_{1}}=\left[\boldsymbol{t}_{i}(\boldsymbol{a}, \boldsymbol{b})\right]_{E_{2}}$ so we must have, in particular, that $\boldsymbol{t}_{i}(\boldsymbol{a}, \boldsymbol{b}) \in \psi(A)$. Now suppose that $E_{1}\left(\boldsymbol{a}, \boldsymbol{a}^{\prime}\right)$, $\rho(\boldsymbol{a}, \boldsymbol{b})$ and $\rho\left(\boldsymbol{a}^{\prime}, \boldsymbol{b}^{\prime}\right)$. Then $[\boldsymbol{b}]_{E_{2}}=\alpha_{A}[\boldsymbol{a}]_{E_{1}}=\alpha_{A}\left[\boldsymbol{a}^{\prime}\right]_{E_{1}}=\left[\boldsymbol{b}^{\prime}\right]_{E_{2}}$. So we have $\boldsymbol{b}, \boldsymbol{b}^{\prime} \in \psi(A)$ and $E_{2}\left(\boldsymbol{b}, \boldsymbol{b}^{\prime}\right)$. Hence $\rho$ defines a natural transformation $\varphi / E_{1} \rightarrow \psi / E_{2}$ which is, therefore, $\alpha$.

\subsection{Local coherence of the functor category}

We will show that our functor category (fp $\mathbf{C}$, Set) is a locally coherent category in the sense that every finitely generated subobject of a finitely presented object is finitely presented. In the abelian context, but not here, this is equivalent to the class of f.p. functors being closed under finite limits. For, we will see that our f.p. functors are closed under finite products but need not be closed under equalisers.

Proposition 2.10. Any finitely generated subfunctor of a finitely presented functor is itself finitely presented.

Proof. Let $F$ be f.p. and $G \subseteq F$ be a f.g. subfunctor. By Corollary 2.8 above $F \cong \varphi / \tilde{\theta}$ for pe-formulas $\varphi$ and $\theta$, and $G \cong \psi / E$ for a pe-formula $\psi$. By Proposition 2.9 the embedding of $G$ in $F$ is defined by a pe-formula $\rho$ :

$$
\psi / E \stackrel{\rho}{\longrightarrow} \varphi / \tilde{\theta}
$$

The functor $G$ is isomorphic to the following subfunctor of $F$ :

$$
\frac{\exists \boldsymbol{z}(\psi(\boldsymbol{z}) \wedge \rho(\boldsymbol{z}, \boldsymbol{x}))}{\tilde{\theta}}
$$

Which is finitely presented.

Corollary 2.11. Any finitely generated subfunctor of a representable functor is defined by a pe-formula.

Proposition 2.12. The category $\mathrm{fp}(\mathrm{fp} \mathbf{C}, \mathbf{S e t})$ is closed under finite products but need not be closed under equalisers.

Proof. Given a positive existential relation $\theta(\boldsymbol{x}, \boldsymbol{y})$, define $\theta_{s}(\boldsymbol{x}, \boldsymbol{y})$ to be the positive existential relation

$$
\theta(\boldsymbol{x}, \boldsymbol{y}) \vee \theta(\boldsymbol{y}, \boldsymbol{x}) \vee \boldsymbol{x}=\boldsymbol{y}
$$


Then $\theta_{s}$ is the symmetric and reflexive closure of $\theta$ and $\tilde{\theta}=\tilde{\theta}_{s}$.

Let $\varphi / \tilde{\theta}$ and $\psi / \tilde{\eta}$ be finitely presented functors. We claim that

$$
\frac{\varphi(\boldsymbol{x})}{\tilde{\theta}\left(\boldsymbol{x}, \boldsymbol{x}^{\prime}\right)} \times \frac{\psi(\boldsymbol{y})}{\tilde{\eta}\left(\boldsymbol{y}, \boldsymbol{y}^{\prime}\right)} \cong \frac{\varphi(\boldsymbol{x}) \wedge \psi(\boldsymbol{y})}{\widetilde{\theta_{s} \wedge \eta_{s}}}
$$

To see this, suppose that $([\boldsymbol{x}],[\boldsymbol{y}])=\left(\left[\boldsymbol{x}^{\prime}\right],\left[\boldsymbol{y}^{\prime}\right]\right)$ in the product. Then $\tilde{\theta}\left(\boldsymbol{x}, \boldsymbol{x}^{\prime}\right)$ and $\tilde{\eta}\left(\boldsymbol{y}, \boldsymbol{y}^{\prime}\right)$. So there is a sequence $\boldsymbol{x}_{0}, \ldots, \boldsymbol{x}_{n} \in \varphi$ and a sequence $\boldsymbol{y}_{0}, \ldots, \boldsymbol{y}_{n} \in$ $\psi$ with $\theta_{s}\left(\boldsymbol{x}, \boldsymbol{x}_{0}\right) \wedge \cdots \wedge \theta_{s}\left(\boldsymbol{x}_{n}, \boldsymbol{x}^{\prime}\right)$ and $\eta_{s}\left(\boldsymbol{y}, \boldsymbol{y}_{0}\right) \wedge \cdots \wedge \eta_{s}\left(\boldsymbol{y}_{n}, \boldsymbol{y}^{\prime}\right)$. The claim follows.

Consider, with notation as above, an equaliser diagram

$$
E \longrightarrow \varphi / \tilde{\theta} \underset{p_{2}}{\stackrel{p_{1}}{\longrightarrow}} \psi / \tilde{\eta}
$$

The natural transformations $p_{1}$ and $p_{2}$ can be expressed by positive existential formulas $\rho_{1}$ and $\rho_{2}$ respectively (by Proposition 2.9). We then have

$$
E=\left\{[\boldsymbol{x}] \in \varphi / \tilde{\theta}: \exists \boldsymbol{y}, \boldsymbol{y}^{\prime} \rho_{1}(\boldsymbol{x}, \boldsymbol{y}) \wedge \rho_{2}\left(\boldsymbol{x}, \boldsymbol{y}^{\prime}\right) \wedge \tilde{\eta}\left(\boldsymbol{y}, \boldsymbol{y}^{\prime}\right)\right\}
$$

So $E$ is the functor given by

$$
\frac{\varphi(\boldsymbol{x}) \wedge \exists \boldsymbol{y}, \boldsymbol{y}^{\prime}\left(\rho_{1}(\boldsymbol{x}, \boldsymbol{y}) \wedge \rho_{2}\left(\boldsymbol{x}, \boldsymbol{y}^{\prime}\right) \wedge \tilde{\eta}\left(\boldsymbol{y}, \boldsymbol{y}^{\prime}\right)\right)}{\tilde{\theta}}
$$

The formula on the top need not be reducible to a first-order formula. Here is an explicit example.

Let $\mathcal{L}$ be the language of rings and let $\theta(x, y)$ be the relation $x=y+1$. Let $E$ be the equaliser of the maps

$$
x=x \underset{2 x}{\stackrel{x}{\longrightarrow}} \frac{x=x}{\tilde{\theta}}
$$

Then $E$ defines the set of $x$ such that $2 x=m+x$ for some $m \in \mathbb{Z}$. So $E(\mathbb{R})=\mathbb{Z}$. If $E$ where reducible to a finitary first-order formula, then this would imply that $\mathbb{Z}$ was definable in $\mathbb{R}$, which is not the case. So $E$ cannot be finitary first-order. This is in contrast to the additive case where the group structure gives finitary first-order definability of such relations. 


\subsection{Coherent functors}

We are now in a position to characterise the category $\operatorname{coh}(\mathrm{fp} \mathbf{C}$, Set) of coherent functors.

Proposition 2.13. $F \in(\mathrm{fp} \mathbf{C}$, Set $)$ is coherent if and only if $F \cong \varphi / \theta$ where $\varphi$ and $\theta$ are positive existential formulas and $\theta$ defines an equivalence relation on $\varphi$.

Proof. $(\Rightarrow)$ Suppose that $F$ is coherent. So in particular $F$ is f.p. and by Corollary $2.8, F \cong \varphi / \tilde{\theta}$. Since $F$ is coherent, $\varphi \times_{F} \varphi$ is f.g. But $\varphi \times_{F} \varphi \cong \tilde{\theta}$ so $\tilde{\theta}$ is f.g. Since $\tilde{\theta} \subseteq \varphi \times \varphi$, the proof of Proposition 2.10 implies that $\tilde{\theta} \cong \eta$ for some pe-formula $\eta$.

$(\Leftarrow)$ Now suppose that $F \cong \varphi / \theta$ where $\varphi$ and $\theta$ are pe-formulas. Let $G$ be a f.g. functor and $f: G \rightarrow F$ a map. By Corollary 2.8 and Proposition 2.9 we can write this as

$$
\frac{\psi}{E} \stackrel{\rho}{\longrightarrow} \frac{\varphi}{\theta}
$$

for some pe-formulas $\psi$ and $\rho$. Let $\sim$ be the equivalence relation on $\psi \times \psi$ defined by

$$
(\boldsymbol{x}, \boldsymbol{y}) \sim\left(\boldsymbol{x}^{\prime}, \boldsymbol{y}^{\prime}\right) \Leftrightarrow E\left(\boldsymbol{x}, \boldsymbol{x}^{\prime}\right) \text { and } E\left(\boldsymbol{y}, \boldsymbol{y}^{\prime}\right)
$$

Let $\gamma(\boldsymbol{x}, \boldsymbol{y})$ be the formula

$$
\exists \boldsymbol{w}, \boldsymbol{z}(\psi(\boldsymbol{x}) \wedge \psi(\boldsymbol{y}) \wedge \rho(\boldsymbol{x}, \boldsymbol{w}) \wedge \rho(\boldsymbol{y}, \boldsymbol{z}) \wedge \theta(\boldsymbol{w}, \boldsymbol{z}))
$$

Then $G \times{ }_{F} G \cong \gamma / \sim$ which is f.g.

We collect together the above proposition and Proposition 2.9 in the following theorem.

Theorem 2.14. The category $\operatorname{coh}(\mathrm{fp} \mathbf{C}$, Set) of coherent functors is equivalent to the category with objects $\varphi / \theta$ where $\varphi$ and $\theta$ are pe-formulas and $\theta$ defines an equivalence relation on $\varphi$, and with maps the pe-definable functions $\varphi / \theta \rightarrow \psi / \eta$ 


\section{$3 \quad$ Finitely presented and coherent sheaves}

\subsection{Extending functors}

In defining localisations of our functor category we will need to make use of the notion of an extension of a functor defined on f.p. objects to the category of all objects. The well-definedness of the extension depends on the following lemma (which has an analogue in the additive case; see, e.g. [2, pp. 4-5], [13, 5.6]). We prove this directly (it is, e.g., contained in $[1,2.26]$ ).

Lemma 3.1. Let $F: \mathrm{fp}(\mathbf{C}) \rightarrow$ Set be a functor defined on the category of finitely presented objects of $\mathbf{C}$. Let $X, Y \in \mathbf{C}$.

(a) If $\left(\left(A_{i}\right)_{i},\left(a_{i k}: A_{i} \rightarrow A_{k}\right)_{i<k}\right)$ and $\left(\left(B_{j}\right)_{j},\left(b_{j l}: B_{j} \rightarrow B_{l}\right)_{j<l}\right)$ are directed systems where $A_{i}, B_{j} \in \mathrm{fp}(\mathbf{C})$ and with $X=\lim _{i} A_{i}=\lim _{j} B_{j}$ then $\lim _{i} F\left(A_{i}\right) \cong \lim _{\longrightarrow} F\left(B_{j}\right)$.

(b) If $X=\lim _{i} X_{i}$ and $Y=\lim _{i} Y_{j}$ and $f: X \rightarrow Y$, then there is a canonically induced map

$$
f_{*}: \underset{i}{\lim } F\left(X_{i}\right) \longrightarrow \underset{j}{\lim } F\left(Y_{j}\right)
$$

which is functorial in the obvious sense.

Proof. (a) Suppose

$$
X=\lim _{i \in I} A_{i}=\underset{j \in J}{\lim _{j \in J}} B_{j}
$$

Since the $A_{i}$ are f.p., for each $i \in I$, there is an $f_{i}: A_{i} \rightarrow B_{k_{i}}$ such that the following diagram commutes.

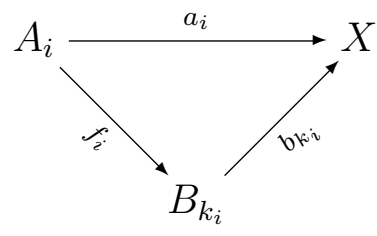


Now consider the following diagram.

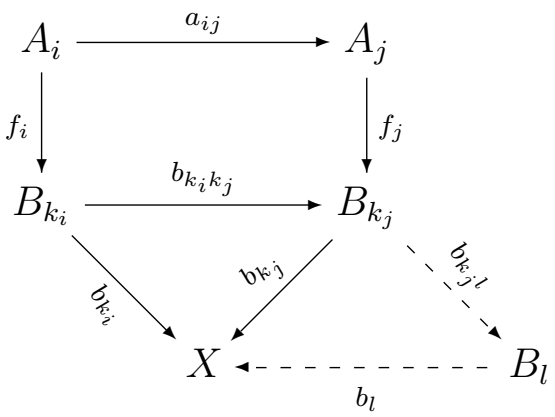

Although the square does not necessarily commute, there will be an $l \in J$ such that $b_{k_{j} l} b_{k_{i} k_{j}} f_{i}=b_{k_{j} l} f_{j} a_{i j}$. This implies that the following diagram is a cocone on the $F\left(A_{i}\right)$.

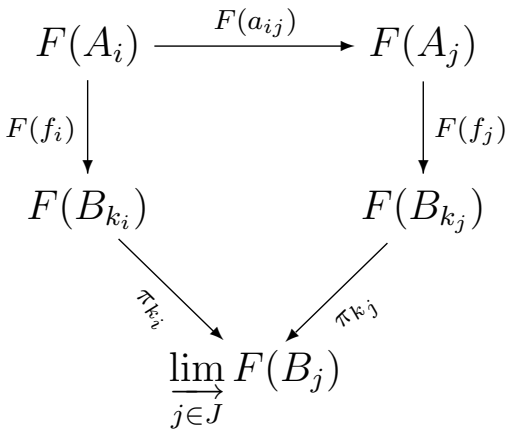

(Here the $\pi_{j}: F\left(B_{j}\right) \rightarrow \underline{\lim }_{l \in J} F\left(B_{l}\right)$ are the canonical maps to the limit. Similarly we write $\sigma_{i}: F\left(\vec{A}_{i}\right) \rightarrow \lim _{k \in I} F\left(A_{k}\right)$.) Hence there is a unique $\rho$ such that the following diagram commutes.

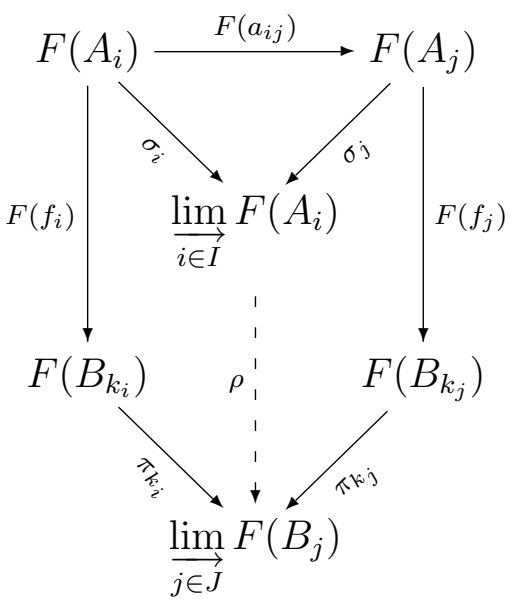


Similarly, for each $j \in J$ there will be a map $g_{j}: B_{j} \rightarrow A_{m_{j}}$ such that $b_{j}=a_{m_{j}} g_{j}$ and one gets a map $\rho^{\prime}: \lim _{\longrightarrow j} F\left(B_{j}\right) \rightarrow \underline{\lim }_{i \in I} F\left(A_{i}\right)$ such that $\rho^{\prime} \pi_{j}=\sigma_{m_{j}} F\left(g_{j}\right)$.

Now consider the following diagram.

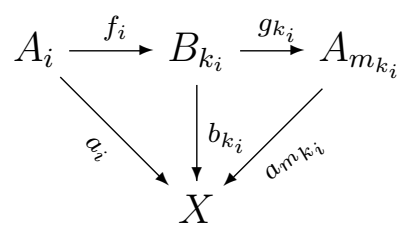

By essential uniqueness, there is an $l_{i} \in I$ such that $a_{k_{i} l_{i}} g_{k_{i}} f_{i}=a_{i l_{i}}$. Then

$$
\begin{aligned}
\rho^{\prime} \rho \sigma_{i} & =\rho^{\prime} \pi_{k_{i}} F\left(f_{i}\right) \\
& =\sigma_{m_{k_{i}}} F\left(g_{k_{i}}\right) F\left(f_{i}\right) \\
& =\sigma_{m_{k_{i}}} F\left(g_{k_{i}} f_{i}\right) \\
& =\sigma_{l_{i}} F\left(a_{k_{i} l_{i}}\right) F\left(g_{k_{i}} f_{i}\right) \\
& =\sigma_{l_{i}} F\left(a_{i l_{i}}\right) \\
& =\sigma_{i}
\end{aligned}
$$

So $\rho^{\prime} \rho$ is the identity on $\lim F\left(A_{i}\right)$. By symmetry, $\rho \rho^{\prime}$ is the identity on $\underset{\lim }{\longrightarrow} F\left(B_{j}\right)$ so $\rho$ is an isomorphism.

(b) The argument for this is similar to that in part (a).

We are now in a position to define the extension of a functor $F \in$ (fp $\mathbf{C}$, Set) to the whole of $\mathbf{C}$. We will denote this extension of $F$ also by $F$, and put for $C \in \mathbf{C}$,

$$
F(C)=\lim _{\longrightarrow} F\left(C_{i}\right)
$$

where $C=\lim C_{i}$ is a directed colimit of f.p. objects. The action of $F$ on maps of $\mathbf{C}$ is given canonically as in the above lemma.

One can also extend a natural transformation $\sigma: F \rightarrow G$ in the category (fp $\mathbf{C}$, Set) to a natural transformation between the extensions by defining, for $C=\lim _{\longrightarrow} C_{i} \in \mathbf{C}$, a directed colimit of f.p. objects,

$$
\sigma_{C}=\lim _{\longrightarrow} \sigma_{C_{i}}
$$

(One may check that this is independent of representation of $C$ as a directed colimit of finitely presented objects.) Any f.p. functor $\varphi / \tilde{\theta}$ in (fp C, Set) extends to the functor $\varphi / \tilde{\theta}: \mathbf{C} \rightarrow$ Set defined by the same formulas and this, 
it is easily checked, commutes with directed colimits. Similarly, a natural transformation given by a pe-formula $\rho$ extends to a functor given by the same formula and which commutes with directed colimits.

\subsection{Definable subcategories and finite type topologies}

Let $\mathbf{A}$ be a small category. Any subfunctor $S$ of $\operatorname{Hom}(A,-)$ may be thought of as a collection of maps with domain $A$ which is closed under composition on the left: i.e. if $f \in S$ and $g$ is a composable map (in the sense that $\operatorname{dom} g=\operatorname{cod} f$ ), then $g f \in S$. We say that $S$ is a (left) ideal of $A$. Given an ideal $S$ of $A$ and a map $f: A \rightarrow B$ in A, one gets an ideal $f_{*}(S)$ of $B$ defined to be $\{g: \operatorname{dom} g=B$ and $g f \in S\}$. A (left) Grothendieck topology $J$ on A is a function which associates to any object $A \in \mathbf{A}$ a set $J(A)$ of left ideals

of $A$. The elements of $J(A)$ are called covers of $A$ or dense subfunctors of $\operatorname{Hom}(A,-)$ and must satisfy the following conditions.

G1 $\operatorname{Hom}(A,-) \in J(A)$ for every $A \in \mathbf{A}$;

G2 If $S \in J(A)$ and $f: A \rightarrow B$ is an arrow of $\mathbf{A}$, then $f_{*}(S) \in$ $J(B)$;

G3 If $S \in J(A)$ and $R \subseteq \operatorname{Hom}(A,-)$ is such that, for any arrow $f \in S, f_{*}(R) \in J(\operatorname{cod} f)$, then $R \in J(A)$.

Fix a subcategory $\mathbf{D}$ of $\mathbf{C}$. We associate to $\mathbf{D}$ a Grothendieck topology $J_{\mathbf{D}}$ on $\operatorname{fp}(\mathbf{C})$ defined by

$$
S \in J_{\mathbf{D}}(A) \quad \Leftrightarrow \quad S(D)=\operatorname{Hom}(A, D) \text { for all } D \in \mathbf{D}
$$

In this definition we are using the extension of $S$ from fp $\mathbf{C}$ to $\mathbf{C}$ as explained in the previous section. One easily verifies that this extension does satisfy the above axioms for a Grothendieck topology.

Conversely, to a given Grothendieck topology $J$ on $\operatorname{fp}(\mathbf{C})$ one can associate a full subcategory $V(J)$ of $\mathbf{C}$ by

$$
D \in V(J) \quad \Leftrightarrow \quad S(D)=\operatorname{Hom}(A, D) \text { for all } A \in \operatorname{fp}(\mathbf{C}) \text { and } S \in J(A)
$$

The following notion (central in the model theory of modules) is needed to describe the "closed" points of this correspondence. 
If $C \subseteq D$ in $\mathbf{C}$ then $C$ is a pure subobject of $D$ if for every commutative diagram

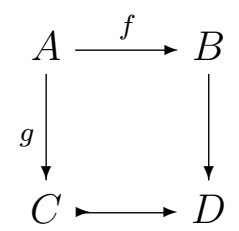

with $A$ and $B$ finitely presented, the map $g$ factors through $f$.

Proposition 3.2. (e.g. $[1,5.15])$ The subobject $C \subseteq D$ is pure if and only if, for every positive existential formula $\varphi(\boldsymbol{x})$ and any tuple $\boldsymbol{c}$ from $C, C=\varphi(\boldsymbol{c})$ if $D \models \varphi(\boldsymbol{c})$.

Proof. It is clearly sufficient to prove the statement for positive primitive formulas.

$(\Rightarrow)$ Suppose that $C \subseteq D$ is pure. Let $\varphi(\boldsymbol{x})$ be the positive primitive formula $\exists \boldsymbol{y} \sigma(\boldsymbol{x}, \boldsymbol{y})$ where $\sigma$ is a conjunction of atomic formulas. Suppose $D \models \varphi(\boldsymbol{c})$ where $\boldsymbol{c}$ is a tuple from $C$. Then there is a tuple $\boldsymbol{d}$ from $D$ such that $D \models \sigma(\boldsymbol{c}, \boldsymbol{d})$. Let $(A, \boldsymbol{a}, \boldsymbol{b})$ be a free realisation of $\sigma$. We get the following commutative diagram, where $\operatorname{Fr}(\boldsymbol{x})$ is the free $\mathcal{L}$-structure generated by a tuple of variables $\boldsymbol{x}$ (e.g. see [1, p. 202]).
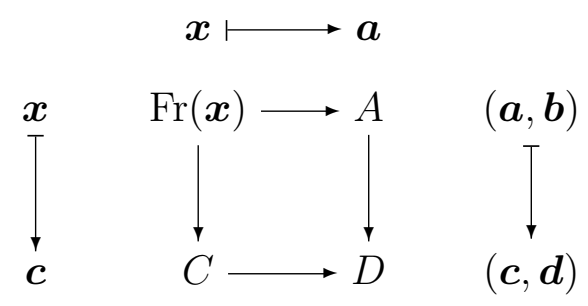

By purity, we get a map $f: A \rightarrow C$ such that $f(\boldsymbol{a})=\boldsymbol{c}$. Since $\boldsymbol{a} \in \varphi(A)$ we must have $\boldsymbol{c} \in \varphi(C)$ as required.

$(\Leftarrow)$ Suppose we have a commutative square

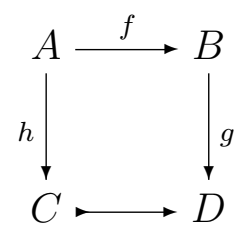

where $A$ and $B$ are finitely presented. Let $\boldsymbol{a}$ be a tuple of generators for $A$ and $\boldsymbol{b}$ a tuple of generators for $B$. Let $\varphi$ be a presentation formula for $B$. 
There is a tuple of terms $\boldsymbol{t}$ such that $f(\boldsymbol{a})=\boldsymbol{t}(\boldsymbol{b})$. Let $\psi(\boldsymbol{x})$ be the positive primitive formula

$$
\exists \boldsymbol{y}(\varphi(\boldsymbol{y}) \wedge \boldsymbol{t}(\boldsymbol{y})=\boldsymbol{x})
$$

Then $D \models \psi(g f(\boldsymbol{a}))$ since $D \models \varphi(g(\boldsymbol{b}))$ and

$$
\boldsymbol{t}(g(\boldsymbol{b}))=g(\boldsymbol{t}(\boldsymbol{b}))=g f(\boldsymbol{a})
$$

Since $g f(\boldsymbol{a})=h(\boldsymbol{a})$ lies in $C$, the hypothesis implies that $C \models \psi(h(\boldsymbol{a}))$. So there is a tuple $\boldsymbol{c} \in \varphi(C)$ such that $\boldsymbol{t}(\boldsymbol{c})=h(\boldsymbol{a})$. By the definition of presentation formula, there is a map $f^{\prime}: B \rightarrow C$ with $f^{\prime}(\boldsymbol{b})=\boldsymbol{c}$. So

$$
h(\boldsymbol{a})=\boldsymbol{t}(\boldsymbol{c})=\boldsymbol{t}\left(f^{\prime}(\boldsymbol{b})\right)=f^{\prime}(\boldsymbol{t}(\boldsymbol{b}))=f^{\prime} f(\boldsymbol{a})
$$

and we see that $h$ factors through $f$ as required.

Following terminology used in the additive case, we will say that a category $\mathbf{D} \subseteq \mathbf{C}$ closed in $\mathbf{C}$ under ultraproducts and pure subobjects is a definable subcategory of $\mathbf{C}$. As the following result shows, this means definable by sentences of a particular sort.

Theorem 3.3. The following are equivalent.

(i) $\mathbf{D}$ is a definable subcategory;

(ii) $\mathbf{D}$ can be axiomatised by a coherent theory.

This result is a direct consequence of a result of Keisler's [11] (also see $[24])$. Recall that a coherent theory is one whose axioms are of the form $\forall \boldsymbol{x}(\varphi(\boldsymbol{x}) \rightarrow \psi(\boldsymbol{x}))$ where $\varphi$ and $\psi$ are positive existential. Keisler's result is that an elementary class of structures is closed under directed colimits if and only if it can be axiomatised by sentences of the form

$$
\forall \boldsymbol{x} \exists \boldsymbol{y} \bigwedge_{i \in I} \bigvee_{j \in J}\left(g_{i j}(\boldsymbol{x}) \rightarrow h_{i j}(\boldsymbol{x}, \boldsymbol{y})\right)
$$

where the $g_{i j}$ and $h_{i j}$ are atomic formulas and $I$ and $J$ are finite. But the above sentence is equivalent to the following collection of coherent sentences

$$
\left\{\forall \boldsymbol{x}\left(\bigwedge_{i \in I^{\prime}} \bigwedge_{j \in J} g_{i j}(\boldsymbol{x}) \rightarrow \exists \boldsymbol{y} \bigwedge_{i \in I^{\prime}} \bigvee_{j \in J} h_{i j}(\boldsymbol{x}, \boldsymbol{y})\right)\right\}_{I^{\prime} \subseteq I}
$$


Now a definable subcategory $\mathbf{D}$ is an elementary class closed under directed colimits. The fact that $\mathbf{D}$ is elementary follows (by [12], Corollary 6.1.16) from the fact that $\mathbf{D}$ is closed under ultraproducts and its complement is closed under ultrapowers (since every structure is purely, even elementarily, embeddable in any of its ultrapowers via the diagonal map). The fact that $\mathbf{D}$ is closed under directed colimits follows from the fact that for any directed system of structures $\left(D_{i}: i \in I\right)$ there is an ultrafilter $\mathcal{U}$ on $I$ and a pure embedding of $\lim _{\longrightarrow} D_{i}$ in $\prod_{i} D_{i} / \mathcal{U}$ (see, e.g., [25]). Hence, Keisler's result gives us that a definable subcategory $\mathbf{D}$ can be axiomatised by a coherent theory. The converse is easy.

Using Theorem 3.3, we see that the class of integral domains forms a definable subcategory of the category of commutative rings: this class is defined by the coherent axiom

$$
\forall x, y(x y=0 \rightarrow x=0 \vee y=0)
$$

We shall say that a Grothendieck topology $J$ is of finite type if every cover has a finitely generated cover below it. In our context this is equivalent to there being a positive existential formula dense below every cover (by Corollary $2,2.11$ ). If $J$ has the stronger property that any cover has a positive primitive formula dense below it, then we shall say it is of finite pp type.

\section{Proposition 3.4.}

(a) If $\mathbf{D}$ is definable, then $J_{\mathbf{D}}$ is of finite type. If in addition $\mathbf{D}$ is closed under finite products, then $J_{\mathbf{D}}$ is of finite $p p$ type.

(b) If $J$ is of finite type, then $V(J)$ is definable. If $J$ is of finite pp type, then $V(J)$ is also closed under products.

Proof. (a) Suppose $\mathbf{D}$ is definable. Let $S \subseteq \operatorname{Hom}(C,-)$ be a cover with respect to $J_{\mathbf{D}}$. Let $\varphi$ be a presentation formula for $C$, so $\operatorname{Hom}(C,-) \cong \varphi$. If $\left\{\varphi_{\lambda}: \lambda \in \Lambda\right\}$ is the set of positive existential formulas below $S$, then clearly

$$
S=\bigcup_{\lambda \in \Lambda} \varphi_{\lambda}
$$

Suppose that none of them is dense. So for each $\lambda$ there is a $D_{\lambda} \in \mathbf{D}$ such that $\varphi_{\lambda}\left(D_{\lambda}\right) \varsubsetneqq \varphi\left(D_{\lambda}\right)$. Let $p$ be the partial type

$$
\left\{\varphi \wedge \neg \varphi_{\lambda}: \lambda \in \Lambda\right\}
$$


Then $p$ is closed under finite conjunctions. Define

$$
\langle\lambda\rangle=\left\{\mu \in \Lambda: \varphi_{\lambda} \subseteq \varphi_{\mu}\right\}
$$

Then

$$
\langle\lambda\rangle \cap\langle\mu\rangle=\langle\nu\rangle
$$

where $\varphi_{\nu}=\varphi_{\lambda} \vee \varphi_{\mu}$. So the set $\{\langle\lambda\rangle: \lambda \in \Lambda\}$ has the finite intersection property and there is an ultrafilter $\mathcal{U}$ containing it. The ultraproduct $D^{*}=$ $\prod_{\lambda} D_{\lambda} / \mathcal{U}$ realises $p$. More concretely, the tuple $\left[\left(\boldsymbol{a}_{\lambda}\right)_{\lambda}\right]$ where $\boldsymbol{a}_{\lambda} \in \varphi\left(D_{\lambda}\right) \backslash$ $\varphi_{\lambda}\left(D_{\lambda}\right)$ is a realisation. To see this, take any $\varphi \wedge \neg \varphi_{\lambda} \in p$. Then

$$
\langle\lambda\rangle \subseteq\left\{\mu \in \lambda: D_{\mu} \models \varphi \wedge \neg \varphi_{\lambda}\left(\boldsymbol{a}_{\mu}\right)\right\}
$$

and so the right hand side is in $\mathcal{U}$. So $D^{*} \models p\left(\left[\left(\boldsymbol{a}_{\lambda}\right)_{\lambda}\right]\right)$. But this means that $S\left(D^{*}\right) \neq \operatorname{Hom}\left(C, D^{*}\right)$ which is a contradiction. So $J_{\mathbf{D}}$ must be of finite type.

Now suppose that in addition $\mathbf{D}$ is closed under finite products. We know from above that any cover $S \subseteq \varphi \cong \operatorname{Hom}(C,-)$ has a dense positive existential formula $\psi$ below it. Write $\psi$ as $\psi_{1} \vee \cdots \vee \psi_{n}$ where each $\psi_{i}$ is positive primitive. Suppose that none of the $\psi_{i}$ is dense. Then for each $i$ there is $D_{i} \in \mathbf{D}$ and $\boldsymbol{a}_{i} \in \varphi\left(D_{i}\right) \backslash \psi_{i}\left(D_{i}\right)$. Let $D=\prod_{i=1}^{n} D_{i}$. Then

$$
\psi(D)=\bigcup_{k=1}^{n}\left(\prod_{i=1}^{n} \psi_{k}\left(D_{i}\right)\right) \subseteq \prod_{i=1}^{n} \varphi\left(D_{i}\right)=\varphi(D)
$$

Since $D \in \mathbf{D}$ and $\psi$ is dense, we must have $\psi(D)=\varphi(D)$. But $\boldsymbol{a}_{k} \in$ $\varphi\left(D_{k}\right) \backslash \psi_{k}\left(D_{k}\right)$ implies that

$$
\left(\boldsymbol{a}_{1}, \ldots, \boldsymbol{a}_{n}\right) \in \prod_{i=1}^{n} \varphi\left(D_{i}\right) \backslash \prod_{i=1}^{n} \psi_{k}\left(D_{i}\right)
$$

for each $k$ which is a contradiction.

(b) Suppose $J$ is a finite type topology. For each $A \in \operatorname{fp} \mathbf{C}$, let $\varphi_{A}$ be a presentation formula for $A$. Now let $T$ be the coherent theory with axioms

$$
\left\{\varphi_{A} \rightarrow \psi: A \in \mathrm{fp} \mathbf{C}, \psi \in J(A)\right\}
$$

Then $V(J)=\operatorname{Mod} T$ (the class of models of $T$ ). By Theorem 3.3, $V(J)$ is definable. 
Suppose now that $J$ is of finite pp type. Let $\psi \subseteq S \subseteq \varphi$ be dense where $\psi$ is positive primitive. Let $D=\prod_{i} D_{i}$ where each $D_{i}$ is in $V(J)$.

$$
\psi(D)=\prod_{i} \psi\left(D_{i}\right)=\prod_{i} \varphi\left(D_{i}\right)=\varphi(D)
$$

so $S(D)=\varphi(D)$ and we must have $D \in V(J)$ as required.

Proposition 3.5. Suppose $\mathbf{D}$ is a definable subcategory. Then $\mathbf{D}=V\left(J_{\mathbf{D}}\right)$.

Proof. Obviously we have $\mathbf{D} \subseteq V\left(J_{\mathbf{D}}\right)$. For the other direction we use Theorem 3.3 .

There is a coherent theory $T^{\prime}$ such that $\mathbf{D}=\operatorname{Mod} T^{\prime}$. Suppose $C \in$ $V\left(J_{\mathbf{D}}\right)$. We want $C \models T^{\prime}$. So consider an arbitrary formula $\forall \boldsymbol{x}(\varphi(\boldsymbol{x}) \rightarrow$ $\psi(\boldsymbol{x}))$ from $T^{\prime}$. We may clearly assume that $\varphi$ is positive primitive and $\psi$ is positive existential. Since $\varphi$ is positive primitive, there is a finitely presented object $A$ and an epimorphism

$$
\operatorname{Hom}(A,-) \stackrel{f}{\longrightarrow} \varphi
$$

Consider the pullback diagram

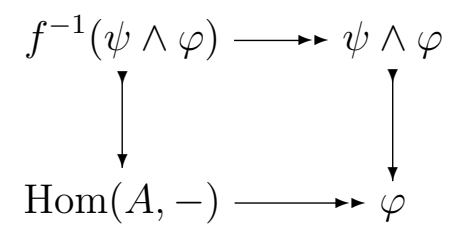

Let $D \in \mathbf{D}$. Then $(\psi \wedge \varphi)(D)=\varphi(D)$, hence $f^{-1}(\psi \wedge \varphi)(D)=\operatorname{Hom}(A, D)$. So $f^{-1}(\psi \wedge \varphi) \in J_{\mathbf{D}}(A)$. But this means $f^{-1}(\psi \wedge \varphi)(C)=\operatorname{Hom}(A, C)$ which implies that $(\psi \wedge \varphi)(C)=\varphi(C)$. So $C \models \forall \boldsymbol{x}(\varphi(\boldsymbol{x}) \rightarrow \psi(\boldsymbol{x}))$ as required.

Recall $([4,6.7])$, or note from the above, that if $\mathbf{D}$ is a definable subcategory closed under finite products, then it is closed under arbitrary products.

In order to prove that there is a bijection between definable subcategories and finite type Grothendieck topologies we will need some sheaf theory.

\subsection{Some general sheaf theory}

Fix a Grothendieck topology $J$ on a skeletally small category A. A presheaf $P \in(\mathbf{A}$, Set $)$ is called a separated presheaf if whenever $A \in \mathbf{A}$ and $f, g$ : 
$\operatorname{Hom}(A,-) \rightarrow P$ are maps such that $f \uparrow_{S}=g\left\lceil_{S}\right.$ for some cover $S \in J(A)$, then $f=g$. A presheaf $F \in(\mathbf{A}$, Set $)$ is called a sheaf if for any object $A \in \mathbf{A}$ and any cover $S \in J(A)$, every map $\alpha: S \rightarrow F$ has a unique extension to a map $\operatorname{Hom}(A,-) \rightarrow F$.

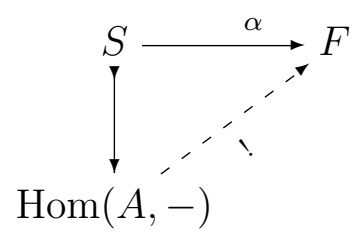

Given a Grothendieck topology $J$ on A, one can form the category of sheaves $\operatorname{Sh}(\mathbf{A}, J)$ which will be a reflexive subcategory of $(\mathbf{A}, \mathbf{S e t})$. So we have an adjoint pair

$$
\operatorname{Sh}(\mathbf{A}, J) \stackrel{i}{\stackrel{i}{\rightleftarrows}}(\mathbf{A}, \text { Set })
$$

where $a$ is the associated sheaf functor and $i$ is the inclusion functor. The functor $a$ is left adjoint to $i$ and commutes with finite limits.

The construction of the associated sheaf is usually done by two applications of Grothendieck's plus functor. This functor takes a presheaf $P$ in (A, Set) to the presheaf $P^{+}$which is defined on objects $C \in \mathbf{A}$ as follows.

$$
P^{+} C=\underset{R \in J(C)}{\lim _{\longrightarrow}} \operatorname{Nat}(R, P)
$$

where the directed colimit is taken over the decreasing family of covers. Note that since a finite intersection of covers is a cover, we do indeed have a directed system over which we can take a limit. $\operatorname{Here} \operatorname{Nat}(R, P)=\operatorname{Hom}(R, P)$ is the set of natural transformations $R \rightarrow P$. The elements of $P^{+} C$ can be represented by pairs $(R, \alpha)$ where $\alpha: R \rightarrow P$ is a natural transformation and $R \in J(C)$. We factor by the equivalence relation

$$
(R, \alpha) \sim(S, \beta) \Leftrightarrow \text { there is a cover } T \subseteq R \cap S \text { s.t. } \alpha\left\lceil_{T}=\beta\left\lceil_{T}\right.\right.
$$

Given a map $f: C \rightarrow C^{\prime}$, the induced map $P^{+} f: P^{+} C \rightarrow P^{+} C^{\prime}$ is defined as follows.

$$
[R, \alpha] \longmapsto\left[f_{*} R, \alpha f^{*}\right]
$$

Here $f^{*}: \operatorname{Hom}\left(C^{\prime},-\right) \rightarrow \operatorname{Hom}(C,-)$ is the induced map and $f_{*} R$ is the cover $\left(f^{*}\right)^{-1} R$ (since an inverse image of a cover is a cover). 
The plus functor takes a natural transformation $\varphi: P \rightarrow Q$ to the natural transformation $\varphi^{+}: P^{+} \rightarrow Q^{+}$which is defined as follows.

$$
\varphi_{C}^{+}[R, \alpha]=[R, \varphi \circ \alpha]
$$

The canonical map $\eta: P \rightarrow P^{+}$is defined as follows.

$$
\eta_{C}(x)=[\operatorname{Hom}(C,-), x]
$$

On the right hand side we identify the element $x \in P(C)$ with the corresponding element of $\operatorname{Nat}(\operatorname{Hom}(C,-), P)$. Clearly $\eta$ is a monomorphism if and only if $P$ is a separated presheaf. For any presheaf $P, P^{+}$is separated. If $P$ is separated, then $P^{+}$is a sheaf. So $P^{++}$is always a sheaf. The associated sheaf functor is equivalent to two applications of the plus functor, so that $a P=P^{++}$.

The following lemmas will be important for us. The first is direct from the definition of $P^{+}$.

Lemma 3.6. For every map $\operatorname{Hom}(A,-) \rightarrow P^{+}$, there is a cover $S \in J(A)$ and a map $S \rightarrow P$ such that the following square commutes.

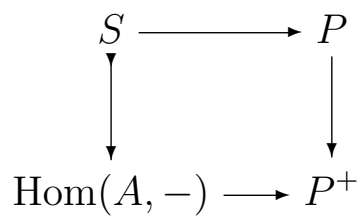

Lemma 3.7. Let $f: a(\operatorname{Hom}(A,-)) \rightarrow a P$ be a map of sheaves. Then there is a cover $S \in J(A)$ such that for every $h \in S$ there is an $x_{h} \in P(C=\operatorname{cod} h)$ such that $a\left(x_{h}\right)=f \circ a\left(h^{*}\right)$ as in the following diagram (which uses the Yoneda identification of $P C$ with $\operatorname{Nat}(\operatorname{Hom}(C,-), P))$.

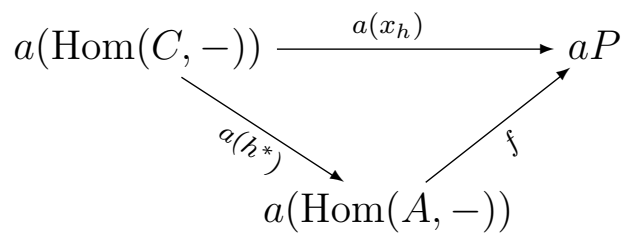

Proof. By applying Lemma 3.6 to the composition

$$
\operatorname{Hom}(A,-) \longrightarrow a(\operatorname{Hom}(A,-)) \stackrel{f}{\longrightarrow} a P
$$


there is a cover $R \in J(A)$ and a map $R \rightarrow P^{+}$s.t. the following diagram commutes.

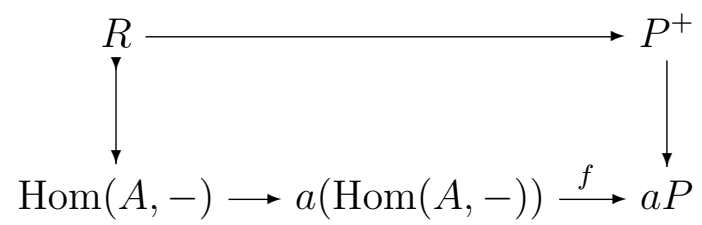

Let $g \in R$ and put $D=\operatorname{cod} g$. Then, again by Lemma 3.6 there is a cover $R_{g} \in J(D)$ and a map $R_{g} \rightarrow P$ such that

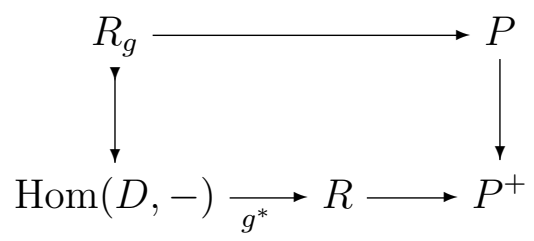

commutes.

Let $S \subseteq \operatorname{Hom}(A,-)$ be defined by $S=\left\{g^{\prime} g: g \in R, g^{\prime} \in R_{g}\right\}$. By G3 of the axioms for a Grothendieck topology $S \in J(A)$. Suppose $h \in S$. Then $h=g^{\prime} g$ for some $g \in R$ and $g^{\prime} \in R_{g}$. The map

$$
\operatorname{Hom}(C=\operatorname{cod} h,-) \stackrel{\left(g^{\prime}\right)^{*}}{\longrightarrow} R_{g} \longrightarrow P
$$

corresponds to an element $x_{h} \in P(C)$ and

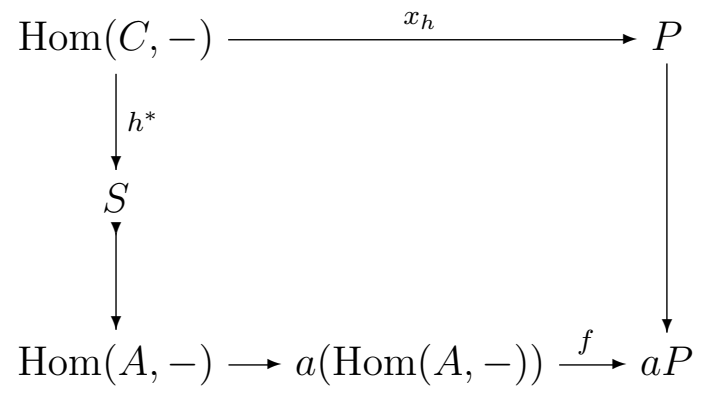

commutes. Hence $a\left(x_{h}\right)=f \circ a\left(h^{*}\right)$ as required.

As described above, the associated sheaf functor $a$ is equivalent to two applications of the plus functor, but it is also equivalent to one application of the "sep-functor" followed by one application of the plus functor. The sep-functor will be relevant to us so we describe it now. 
We define an equivalence relation on $P(C)$ by

$$
x \sim y \quad \Leftrightarrow \exists S \in J(C) \text { s.t. } x \uparrow_{S}=y \uparrow_{S}
$$

Define $P_{\text {sep }} C=P(C) / \sim$. A map $f: C \rightarrow C^{\prime}$ induces a map $f_{*}: P_{\text {sep }}(C) \rightarrow$ $P_{\text {sep }}\left(C^{\prime}\right)$ in the obvious way. This association is functorial and so $P_{\text {sep }}$ is a well defined element of (A, Set). Moreover, the map

$$
P \longmapsto P_{\text {sep }}
$$

is also functorial as one can check (the induced maps are the obvious ones). There is a canonical epimorphism $\sigma: P \rightarrow P_{\text {sep }}$.

Lemma 3.8. $P_{\text {sep }}$ is separated.

Proof. Let $S \in J(C)$ and let $x, y \in P(C)$ with $\bar{x}, \bar{y}$ the corresponding equivalence classes in $P_{\text {sep }} C$. Suppose that $\bar{x} \uparrow_{S}=\bar{y} \uparrow_{S}$. So for each $f \in S$, there is an $R_{f} \in J(\operatorname{cod} f)$ such that

$$
f_{*}(x) \uparrow_{R_{f}}=f_{*}(y)\left\lceil R_{f}\right.
$$

Let $S^{\prime}$ be the set

$$
\left\{g f: f \in S, g \in R_{f}\right\}
$$

Then $S^{\prime} \in J(C)$ (by Grothendieck topology axiom G3) and $x \uparrow_{S^{\prime}}=y \uparrow_{S^{\prime}}$. So $\bar{x}=\bar{y}$ and $P_{\text {sep }}$ is separated as required.

Since $P_{\text {sep }}$ is separated, the canonical map $\eta: P_{\text {sep }} \rightarrow\left(P_{\text {sep }}\right)^{+}$is a monomorphism and $\left(P_{\text {sep }}\right)^{+}$is a sheaf. Since $\sigma$ is universal from $P$ to separated presheaves and $\eta$ is universal from $P$ to sheaves (these statements are easily verified), it follows that $(-)^{+} \circ(-)_{\text {sep }}=a$. We have the following picture.

$$
P \longrightarrow P_{\text {sep }} \longrightarrow a P
$$

Let $P$ be any presheaf. A subobject $P^{\prime} \subseteq P$ is dense (w.r.t. the ambient Grothendieck topology $J$ ) if for any representable functor $\operatorname{Hom}(A,-)$ and any map $f: \operatorname{Hom}(A,-) \rightarrow P$, the inverse image $f^{-1}\left(P^{\prime}\right)$ is in $J(A)$. For any presheaf $P$, we use the notation $\mathcal{D}(P)$ for its set of dense subobjects. This set is closed under finite intersections so forms a downward directed family.

Lemma 3.9.

$$
\operatorname{Nat}(a P, a Q) \cong \underset{P^{\prime} \in \mathcal{D}(P)}{\lim _{\longrightarrow}} \operatorname{Nat}\left(P^{\prime}, Q_{\text {sep }}\right)
$$


Proof. First note that $\operatorname{Nat}(a P, a Q) \cong \operatorname{Nat}(P, a Q)$. Define the map

$$
\Phi: \operatorname{Nat}(P, a Q) \longrightarrow \underset{P^{\prime} \in \mathcal{D}(P)}{\lim _{\longrightarrow}} \operatorname{Nat}\left(P^{\prime}, Q_{\text {sep }}\right)
$$

by

$$
\Phi(\alpha)=\left[\alpha^{-1} Q_{\text {sep }}, \alpha\left(\text {, rather } \alpha \uparrow \alpha^{-1} Q_{\text {sep }}\right)\right]
$$

In the other direction, we define the map

$$
\Psi: \underset{P^{\prime} \in \mathcal{D}(P)}{\lim _{\longrightarrow}} \operatorname{Nat}\left(P^{\prime}, Q_{\text {sep }}\right) \longrightarrow \operatorname{Nat}(P, a Q)
$$

by

$$
\Psi\left[P^{\prime}, \alpha\right]_{C}(x)=\left[x^{-1} P^{\prime}, \alpha \circ x\right]
$$

Note that the morphism $\alpha \circ x: x^{-1} P^{\prime} \rightarrow Q_{\text {sep }}$ has a unique extension to a morphism $\operatorname{Hom}(C,-) \rightarrow a Q$, that is, to an element of $a Q(C)$. It is easily checked that, since $Q_{\text {sep }}$ is separated, $\Psi$ is well defined. We claim that $\Psi=$ $\Phi^{-1}$.

We first show that $\Psi \circ \Phi=$ id.

$$
\begin{aligned}
\Psi(\Phi(\alpha))_{C}(x) & =\Psi\left[\alpha^{-1} Q_{\mathrm{sep}}, \alpha\right]_{C}(x) \\
& =\left[x^{-1}\left(\alpha^{-1} Q_{\mathrm{sep}}\right), \alpha \circ x\right]
\end{aligned}
$$

Write $R=x^{-1}\left(\alpha^{-1} Q_{\text {sep }}\right)$. Suppose that $\alpha_{C}(x)=[S, \beta]$. We want to show that there is a cover $T \subseteq R \cap S$ such that $\alpha \circ x \uparrow_{T}=\beta \uparrow_{T}$.

For any $f: C \rightarrow D$ in $R$,

$$
\begin{aligned}
(\alpha \circ x)(f) & =\alpha_{D}\left(f_{*}(x)\right) \\
& =f_{*}\left(\alpha_{C}(x)\right) \\
& =f_{*}[S, \beta] \\
& =\left[f_{*} S, \beta f^{*}\right]
\end{aligned}
$$

But we know that $(\alpha \circ x)(f)$ is the element $[\operatorname{Hom}(D,-),(\alpha \circ x)(f)] \in a Q$. So there is a cover $S_{f} \subseteq f_{*} S$ such that

$$
\beta f^{*} \Upsilon_{S_{f}}=(\alpha \circ x)(f) \Upsilon_{S_{f}}
$$

So setting

$$
T=\left\{g f: f \in R, g \in S_{f}\right\}
$$


we see that $T$ is a cover such that $\alpha \circ x \uparrow_{T}=\beta \uparrow_{T}$ as required.

We now show that $\Phi \circ \Psi=$ id. Let $\beta=\Psi\left[P^{\prime}, \alpha\right]$, so

$$
\beta_{C}(x)=\left[x^{-1} P^{\prime}, \alpha \circ x\right]
$$

Then we have

$$
\Phi(\beta)=\left[\beta^{-1} Q_{\mathrm{sep}}, \beta\right] .
$$

Now, $P^{\prime} \subseteq \beta^{-1} Q_{\text {sep }}$ since if $x \in P^{\prime} C$ then $\beta_{C}(x)=[\operatorname{Hom}(C,-), \alpha \circ x] \in Q_{\text {sep }}$. Clearly $\beta \uparrow_{P^{\prime}}=\alpha$ and the result follows.

\subsection{Geometric morphisms, points, and coherent toposes}

The class of all toposes can be made into a category (actually it is a 2category, see $[3$, Vol. 1, 7.1]). A map of toposes $\varphi: \mathcal{E} \rightarrow \mathcal{F}$ is defined to be a geometric morphism, meaning an adjoint pair

$$
\mathcal{E} \underset{\varphi^{*}}{\stackrel{\varphi_{*}}{\longrightarrow}} \mathcal{F}
$$

where $\varphi^{*}$ is left adjoint to $\varphi_{*}$ and $\varphi^{*}$ is left exact (i.e. commutes with finite limits). Composition can be defined by composing the adjoints (see [14, p. 101]). A point $p$ of a topos $\mathcal{E}$ is a geometric morphism $p:$ Set $\rightarrow \mathcal{E}$. A topos $\mathcal{E}$ is said to have enough points if for any two subobjects $A, B$ of a given object $E \in \mathcal{E}$, we have that

$$
A \subseteq B \quad \Leftrightarrow \quad p^{*}(A) \subseteq p^{*}(B) \text { for all points } p: \text { Set } \rightarrow \mathcal{E}
$$

A site is a pair $(\mathbf{A}, J)$ consisting of a small category $\mathbf{A}$ with a Grothendieck topology $J$. The site is called coherent if $J$ is of finite type. So if $\mathbf{C}$ is an LFP category and $\mathbf{D} \subseteq \mathbf{C}$ a definable subcategory, then the site ( $f p \mathbf{C}, J_{\mathbf{D}}$ ) is coherent (by Proposition 3.4(a)). A coherent topos is a topos which can be realised as the category of sheaves for a coherent site. The following theorem is proved in $[15, \mathrm{IX}, \S 11]$.

Theorem 3.10 (Deligne). A coherent topos has enough points.

Suppose that $p$ is a point of the presheaf topos (A, Set). Since every presheaf is a colimit of representables, and $p^{*}$, as a left adjoint, commutes 
with all colimits, $p^{*}$ is determined by its action on those representables. So $p^{*}$ is determined by its composition with the Yoneda embedding

$$
\mathbf{A}^{\mathrm{op}} \longrightarrow(\mathbf{A}, \text { Set })
$$

Conversely, any functor $F: \mathbf{A}^{\mathrm{op}} \rightarrow$ Set determines a functor $(\mathbf{A}$, Set $) \rightarrow$ Set by associating to a presheaf $P \in(\mathbf{A}$, Set $)$ the set

$$
\frac{\coprod_{A \in \mathbf{A}} F(A) \times P(A)}{\sim}
$$

where $(x u, y) \sim(x, u y)$ for all $u: A \rightarrow B$ in $A, x \in F(B)$ and $y \in P(A)$ (here $x u$ is defined to be $F(u)(x)$ and $u y$ is $P(u)(y)$ ). This functor has an obvious action on maps and is denoted by $F \otimes_{\mathbf{A}}-$ (see [15, VII, §2]). Like the tensor product for modules, it has a right adjoint Set $\rightarrow(\mathbf{A}$, Set $)$ given by

$$
X \longmapsto \operatorname{Hom}(F(-), X)
$$

So $F$ determines a geometric morphism Set $\rightarrow(\mathbf{A}$, Set $)$ if and only if $F \otimes_{\mathbf{A}}-$ is left exact. A functor $F: \mathbf{A}^{\mathrm{op}} \rightarrow$ Set such that $F \otimes_{\mathbf{A}}-$ is left exact is called a flat functor. Now every functor $F \in\left(\mathbf{A}^{\mathrm{op}}\right.$, Set $)$ is a colimit of representable functors in a canonical way: the indexing category is the category of elements of $F$ whose objects are pairs $(A, x)$ where $A \in \mathbf{A}$ and $x \in F(A)$ and whose maps $(A, x) \rightarrow(B, y)$ are those maps $f: A \rightarrow B$ such that $F(f)(y)=x$. Let $U$ be the forgetful functor from the category of elements of $F$ to $\mathbf{A}$. Then the maps $\operatorname{Hom}(-, U(A, x)) \rightarrow F$ corresponding to $x \in F(A)$ give a cocone on $F$. This cocone is in fact a colimit and will be said to be canonical. If the category of elements of $F$ is a filtered category (see [1, p. 13]), then we can express $F$ canonically as a filtered colimit of representable functors. It turns out that this property is equivalent to flatness: $F$ is flat if and only if its category of elements is filtered ([15, VII, Thm 9.1]).

We have seen that a flat functor $F \in\left(\mathbf{A}^{\mathrm{op}}\right.$, Set $)$ determines a point of (A, Set). In fact, all points of (A, Set) arise in this way (see [15, VII, Thm $5.2])$. Now, suppose that $J$ is a Grothendieck topology on $\mathbf{A}$ and $p$ is a point of $\operatorname{Sh}(\mathbf{A}, J)$. The inclusion of sheaves in presheaves has a left exact left adjoint and so gives a geometric morphism

$$
\operatorname{Sh}(\mathbf{A}, J) \rightarrow(\mathbf{A}, \mathbf{S e t})
$$

By composition with this arrow, the point $p$ gives a point of $(\mathbf{A}$, Set $)$ and so must be induced by a flat functor $F: \mathbf{A}^{\mathrm{op}} \rightarrow$ Set. The functor $F$ then 
has the property that, whenever $S \subseteq \operatorname{Hom}(A,-)$ is a cover, the induced monomorphism $F \otimes_{\mathbf{A}} S \rightarrow F \otimes_{\mathbf{A}} \operatorname{Hom}(A,-)$ is an isomorphism. Such a functor is said to be $J$-continuous or simply continous if the context is clear. Conversely, given a flat $J$-continuous functor $F$, the induced point of $(\mathbf{A}$, Set) factors through the sheaf category and gives a point of $\operatorname{Sh}(\mathbf{A}, J)$. Every point of $\operatorname{Sh}(\mathbf{A}, J)$ arises in this way (see [15, VII, Lemma 5.3]).

Lemma 3.11. Let $J$ be a Grothendieck topology on $\mathrm{fp} \mathbf{C}$. A functor $F$ : $(\mathrm{fp} \mathbf{C})^{\mathrm{op}} \rightarrow$ Set is flat and J-continous if and only if it is of the form $\operatorname{Hom}(-, D)$ for some object $D \in V(J)$.

Proof. Firstly, suppose that $D \in V(J)$. The functor $\operatorname{Hom}(-, D) \otimes_{\mathrm{fp}} \mathbf{C}-$ is isomorphic to $\operatorname{ev}_{D}$ (the "evaluation at $D$ " functor). That is, if $P \in(\mathrm{fp} \mathbf{C}, \mathbf{S e t})$, then $(\operatorname{Hom}(-, D) \otimes P) \cong P(D)$ in a natural way. In an LFP category, directed colimits commute with finite limits (see [1, Prop 1.59]). Since $\mathbf{C}$ is LFP and the extension of $P$ to $\mathbf{C}$ is computed via directed colimits (see Lemma 3.1), we have that $\operatorname{ev}_{D}$ commutes with finite limits. So $\operatorname{Hom}(-, D) \otimes-$ is left exact which implies that $\operatorname{Hom}(-, D)$ is flat. Now, if $S \subseteq \operatorname{Hom}(C,-)$ is a cover, then $\operatorname{Hom}(-, D) \otimes S \rightarrow \operatorname{Hom}(-, D) \otimes \operatorname{Hom}(C,-)$ is the inclusion $S(D) \subseteq \operatorname{Hom}(C, D)$. Since $D \in V(J)$, this inclusion is an equality. Hence $\operatorname{Hom}(-, D)$ is continuous.

Conversely, suppose that $F$ is a continous flat functor $(\mathrm{fp} \mathbf{C})^{\text {op }} \rightarrow$ Set. Since $F$ is flat, we can write $F=\lim _{i} \operatorname{Hom}\left(-, A_{i}\right)$ as a filtered colimit of representables. Since, for any $C \in \mathrm{fp} \overrightarrow{\mathbf{C}}$ we have that $\operatorname{Hom}(C,-)$ commutes with filtered colimits in $\mathbf{C}$ (see $[1, \operatorname{Cor} 1.5]$ ), we have that $F \cong \operatorname{Hom}\left(-, \lim _{\longrightarrow} A_{i}\right)$. Let $D=\lim _{i} A_{i}$. Then $(F \otimes-) \cong \mathrm{ev}_{D}$ and for any object $C \in \mathrm{fp} \mathbf{C} \overrightarrow{\text { and }}$ any cover $S \subseteq \overrightarrow{\operatorname{Hom}}^{2}(C,-)$, we have that $S D=\operatorname{Hom}(C, D)$ since $F$ is continous. So $D \in V(J)$ as required.

We can use Deligne's theorem and the above lemma to prove the following.

Proposition 3.12. Let $J$ be a finite type topology on $\mathrm{fp} \mathbf{C}$. Then $J=J_{V(J)}$.

Proof. We clearly have that $J \subseteq J_{V(J)}$ so we need only establish that $J_{V(J)} \subseteq$ $J$. Let $C \in \mathrm{fp} \mathbf{C}$ and $S \in J_{V(J)}(C)$. Let $a:(\mathrm{fp} \mathbf{C}$, Set $) \rightarrow \operatorname{Sh}(\mathrm{fp} \mathbf{C}, J)$ be the associated sheaf functor. Consider the inclusion $a S \subseteq a(\operatorname{Hom}(C,-))$. If $p$ is a point of $\operatorname{Sh}(\mathrm{fp} \mathbf{C}, J)$, by Lemma 3.11 and the description of points of $\operatorname{Sh}($ fp $\mathbf{C}, J)$, there is an object $D \in V(J)$ such that for any presheaf $P$, we have $p^{*}(a P) \cong(\operatorname{Hom}(-, D) \otimes P) \cong P D$. So we have that $p^{*}(a S)=S(D)=$ $\operatorname{Hom}(C, D)=p^{*}(a \operatorname{Hom}(C,-))$ since $S \in J_{V(J)}(C)$ and $D \in V(J)$. Since 
$J$ is of finite type, the topos $\operatorname{Sh}(\operatorname{fp} \mathbf{C}, J)$ has enough points (by Deligne's Theorem). Hence we have that $a S=a(\operatorname{Hom}(C,-))$. But this implies that $S \in J(C)$. So we have that $J_{V(J)}=J$ as required.

Combining Propositions 3.5 and 3.12 we get the following corollary which is completely analogous to the additive case (see $[22,13.3 .6]$ ).

Corollary 3.13. There is a bijection between definable subcategories of $\mathbf{C}$ and finite type topologies on $\mathrm{fp} \mathbf{C}$.

That Deligne's Theorem could be used to prove Proposition 3.12 was suggested to us by Tibor Beke.

\subsection{Finitely presented sheaves}

Let $J$ be a Grothendieck topology on $\mathrm{fp} \mathbf{C}$. We have an adjunction

$$
\operatorname{Sh}(\text { fp } \mathbf{C}, J) \underset{a}{\stackrel{i}{\rightleftarrows}}(\text { fp } \mathbf{C}, \text { Set })
$$

as before, where the associated sheaf functor a commutes with finite limits. Note that a monomorphism $f$ is a dense embedding of presheaves if and only if $a(f)$ is invertible in the category of sheaves. The next result is general: if a functor with a left adjoint commutes with directed colimits then its left adjoint preserves finitely presented objects.

Lemma 3.14. Suppose $i$ commutes with directed colimits. Then for any finitely presented (resp. finitely generated) functor $P$ in the presheaf category (fp C, Set), aP is finitely presented (resp. finitely generated) in the category of sheaves.

Proof. Let $P$ be a f.p. (resp. f.g.) presheaf and let $\underset{\lim }{\longrightarrow} G_{i}$ be a directed colimit (resp. directed union) of sheaves. Note that since $i$ preserves monomorphisms, directed unions are taken to directed unions. Then:

$$
\begin{aligned}
& \operatorname{Nat}\left(a P, \varliminf_{\lim _{i}} G_{i}\right) \cong \operatorname{Nat}\left(P, i\left(\underline{\lim }_{\longrightarrow} G_{i}\right)\right) \cong \operatorname{Nat}\left(P, \underline{\lim }_{\longrightarrow} i G_{i}\right) \\
& \cong \lim _{\longrightarrow} \operatorname{Nat}\left(P, i G_{i}\right) \cong \lim _{\longrightarrow} \operatorname{Nat}\left(a P, G_{i}\right)
\end{aligned}
$$

Proposition 3.15. The topology $J$ is of finite type if and only if the inclusion functor $i$ commutes with directed colimits. 
Proof. $(\Rightarrow)$ Suppose $J$ is of finite type. Let $\left\{F_{i}\right\}_{i}$ be directed system of sheaves and let $\lim _{\longrightarrow} F_{i}$, with limit maps $f_{k}: F_{k} \rightarrow \lim _{\longrightarrow} F_{i}$ be their directed colimit as presheaves. Let $\psi$ be a finitely presented cover of an object $A \in$ $\mathrm{fp}(\mathbf{C})$. Consider a natural transformation $\alpha: \psi \rightarrow \varliminf_{\longrightarrow} F_{i}$. Since $\psi$ is f.p. $\alpha$ factors essentially uniquely through some $F_{k}$ as $f_{k} \beta$, as shown. But since $F_{k}$ is a sheaf, $\beta$ extends uniquely to a natural transformation $\rho$ on $\operatorname{Hom}(A,-)$ as in the following diagram.

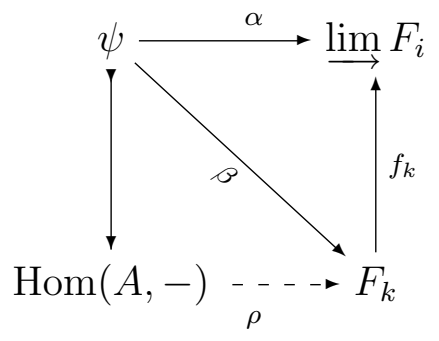

Clearly $f_{k} \rho$ is the unique extension of $\alpha$ to $\operatorname{Hom}(A,-)$, so $\lim F_{i}$ is a sheaf. $(\Leftarrow)$ Now suppose that $i$ commutes with directed colimits. Let $S \subseteq$ $\operatorname{Hom}(A,-)$ be a cover. Write

$$
S=\bigcup_{\lambda} \varphi_{\lambda}
$$

as a directed union of finitely presented subobjects. Since $a$ is a left adjoint it commutes with directed colimits. It also commutes with finite limits and so preserves monomorphisms. Hence

$$
a(\operatorname{Hom}(A,-))=a S=\bigcup_{\lambda} a\left(\varphi_{\lambda}\right)
$$

is a directed union in the sheaf category. Since $i$ commutes with directed colimits, we have, by Lemma 3.14 , that $a(\operatorname{Hom}(A,-))$ is finitely presented in the sheaf category. So $a(\operatorname{Hom}(A,-))=a\left(\varphi_{\lambda}\right)$ for some $\lambda$. But this means that $\varphi_{\lambda} \subseteq \operatorname{Hom}(A,-)$ is dense. This proves that $J$ is of finite type.

Proposition 3.16. Let $J$ be a finite type topology.

(a) If $F$ is a finitely generated sheaf, there is a finitely generated presheaf $P$ such that $F \cong a P$;

(b) IF $F$ is a finitely presented sheaf, then there is a finitely presented presheaf $P$ such that $F \cong a P$. 
Proof. (a) By Lemma 3.14 if $J$ is a finite type topology, then $\operatorname{Sh}(\mathrm{fp} \mathbf{C}, J)$ is an LFP topos with a generating set

$$
\{a(\operatorname{Hom}(A,-)): A \in \mathrm{fp} \mathbf{C}\}
$$

of finitely presented objects. By Proposition 2.1 there is an epimorphism

$$
\coprod_{i} a\left(\operatorname{Hom}\left(A_{i},-\right)\right) \longrightarrow F \cong a F
$$

where the coproduct is finite. By Lemma 3.7, for each component $a\left(\operatorname{Hom}\left(A_{i},-\right)\right) \rightarrow$ $a F$ there is a cover $S_{i}$ of $A_{i}$ such that for each $C \in \mathrm{fp} \mathbf{C}$ and $h \in S_{i} C$, the composition

$$
a(\operatorname{Hom}(C,-)) \longrightarrow a\left(\operatorname{Hom}\left(A_{i},-\right)\right) \longrightarrow a F
$$

is of the form $a\left(f_{i, h}\right)$ for some $f_{i, h}: \operatorname{Hom}(C,-) \rightarrow F$. Since the topology $J$ is of finite type, we can assume that $S_{i}$ is finitely presented, so there are finitely many $C_{k}$ and $h_{k} \in S_{i} C_{k}$ and an epimorphism

$$
\coprod_{k} \operatorname{Hom}\left(C_{k},-\right) \longrightarrow S_{i}
$$

This gives an epimorphism

$$
\coprod_{k} a\left(\operatorname{Hom}\left(C_{k},-\right)\right) \longrightarrow a\left(\operatorname{Hom}\left(A_{i},-\right)\right)
$$

Given all this, and since $a$ commutes with coproducts, it is clear that we can choose the $A_{i}$ such that our epimorphism

$$
\coprod_{i} a\left(\operatorname{Hom}\left(A_{i},-\right)\right) \longrightarrow a F
$$

is of the form $a(f)$ for some $f: \amalg_{i} \operatorname{Hom}\left(A_{i},-\right) \rightarrow F$. Put $P=\operatorname{im} f$. Then $P$ is f.g. and $a P \cong a F \cong F$.

(b) Now suppose that $F$ is f.p., so there is a presentation for $F$ of the form

$$
\coprod_{i} a\left(\operatorname{Hom}\left(A_{i},-\right)\right) \underset{q}{\stackrel{p}{\rightleftarrows}} \coprod_{j} a\left(\operatorname{Hom}\left(B_{j},-\right)\right) \longrightarrow F
$$

where all coproducts are finite. The argument of part (a) applies here to give us that, when the $A_{i}$ are chosen suitably, $p=a(f)$ and $q=a(g)$ for maps

$$
\coprod_{i} \operatorname{Hom}\left(A_{i},-\right) \underset{g}{\stackrel{f}{\rightleftarrows}} \coprod_{j} \operatorname{Hom}\left(B_{j},-\right)
$$


Since $a$ commutes with colimits, $F \cong a P$ where $P$ is the, clearly finitely presented, coequaliser of the above diagram.

For the remainder of this section, let $J=J_{\mathbf{D}}$ for a definable subcategory $\mathbf{D} \subseteq \mathbf{C}$. In this case we have the following neat characterisation of dense subobjects of presheaves (involving the extension of functors from fp $\mathbf{C}$ to $\mathbf{C}$ defined earlier).

Lemma 3.17. $P^{\prime} \subseteq P$ is a dense inclusion of presheaves if and only if $P^{\prime}(D)=P(D)$ for all $D \in \mathbf{D}$.

Proof. Let $D \in \mathbf{D}$ and $x \in P(D)$. Then there is a f.p. object $C \in \mathbf{C}$, an element $y \in P(C)$ and a map $f: C \rightarrow D$ s.t. $f_{*}(y)=x$. Since $P^{\prime}$ is dense in $P$, we have that $\left(y^{-1} P^{\prime}\right)(D)=\operatorname{Hom}(C, D)$. So $f_{*}(y) \in P^{\prime}(D)$, i.e. $x \in P^{\prime}(D)$ as required.

For the converse, assume $P^{\prime}(D)=P(D)$ for all $D \in \mathbf{D}$. Let $A \in \mathrm{fp} \mathbf{C}$, and $f: \operatorname{Hom}(A,-) \rightarrow P$ a natural transformation. Then our assumption implies that $\left(f^{-1} P^{\prime}\right)(D)=f^{-1}\left(P^{\prime} D\right)=f^{-1}(P D)=\operatorname{Hom}(A, D)$. So by definition of $J_{\mathbf{D}}$, we have that $f^{-1} P^{\prime}$ is dense in $\operatorname{Hom}(A,-)$. This implies that $P^{\prime}$ is dense in $P$.

We are now in a position to prove a characterisation result for the category of finitely presented sheaves $\operatorname{fp}\left(\operatorname{Sh}\left(\mathrm{fp} \mathbf{C}, J_{\mathbf{D}}\right)\right)$ where $\mathbf{D} \subseteq \mathbf{C}$ is a definable subcategory. Let $P$ and $Q$ be finitely presented presheaves. So $P \cong \varphi / \tilde{\theta}$ and $Q \cong \psi / \tilde{\eta}$ say. Let $E \subseteq \psi \times \psi$ be the functorial equivalence relation defined by

$$
E(C)=\left\{(\boldsymbol{x}, \boldsymbol{y}) \in \psi(C) \times \psi(C): \exists R \in J_{\mathbf{D}}(C) \text { s.t. } \forall g \in R, \tilde{\eta}(g(\boldsymbol{x}), g(\boldsymbol{y}))\right\}
$$

It is clear that $Q_{\text {sep }}=\psi / E$.

Note that $\tilde{\eta} \subseteq E$ is dense. To see this, let $C \in \mathrm{fp} \mathbf{C}$ and suppose that $(\boldsymbol{x}, \boldsymbol{y}) \in E(C)$. We need to show that $(\boldsymbol{x}, \boldsymbol{y})^{-1} \tilde{\eta}$ is a cover of $C$. That is, $S=\{f \in \operatorname{Hom}(C,-): \tilde{\eta}(f(\boldsymbol{x}), f(\boldsymbol{y}))\}$ is in $J_{\mathbf{D}}(C)$. Let $f: C \rightarrow D$ be a map with $D \in \mathbf{D}$. We know there is an $R \in J_{\mathbf{D}}(C)$ such that for all $g \in R$, $\tilde{\eta}(g(\boldsymbol{x}), g(\boldsymbol{y}))$ holds. But since $R(D)=\operatorname{Hom}(C, D)$ we have $f \in R(D)$ and so $\tilde{\eta}(f(\boldsymbol{x}), f(\boldsymbol{y}))$ which implies $f \in S$. This shows that $S$ is a cover of $C$ and that $\tilde{\eta} \subseteq E$ is dense.

We claim that $E$ is in fact the closure of $\tilde{\eta}$ in $\psi \times \psi$ (that is, $E$ is the largest subobject of $\psi \times \psi$ in which $\tilde{\eta}$ is dense). Let $E \subseteq F \subseteq \psi \times \psi$ be such that $E(D)=F(D)$ for all $D \in \mathbf{D}$. We want to show that $E=F$. 
So take $(\boldsymbol{x}, \boldsymbol{y}) \in F C$. This element defines a natural transformation $\alpha$ : $\operatorname{Hom}(C,-) \rightarrow F$ and we can take the pullback as in the following diagram.

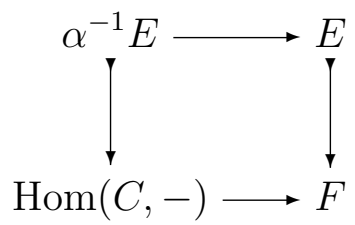

Since $E \subseteq F$ is dense, $\alpha^{-1} E$ is in $J_{\mathbf{D}}(C)$. For all $f \in \alpha^{-1} E, \alpha(f)=f_{*}(\boldsymbol{x}, \boldsymbol{y})=$ $(f(\boldsymbol{x}), f(\boldsymbol{y})) \in E(\operatorname{cod} f)$. So there is a cover $R_{f}$ such that for all $g \in R_{f}$, $\tilde{\eta}(g f(\boldsymbol{x}), g f(\boldsymbol{y}))$. So putting

$$
T=\left\{g f: f \in \alpha^{-1} E, g \in R_{f}\right\}
$$

we have that $T \in J_{\mathbf{D}}(C)$ and for all $h \in T, \tilde{\eta}(h(\boldsymbol{x}), h(\boldsymbol{y}))$. So $(\boldsymbol{x}, \boldsymbol{y}) \in E(C)$ which means that $E=F$. This shows that $E$ is indeed the closure of $\tilde{\eta}$ in $\psi \times \psi$.

Now let us write $E$ as the directed union of positive existential formulas $\eta_{\lambda}$ such that $\eta \subseteq \eta_{\lambda} \subseteq E$. Then $\psi / E=\lim _{\lambda} \psi / \tilde{\eta}_{\lambda}$ as a directed colimit of finitely presented functors. Since $J_{\mathbf{D}}$ is a finite type topology, any dense subobject $P^{\prime}$ of $P$ will have a dense f.p. object (of the form given by Corollary 2.11) below it. This can be seen from the following diagram.

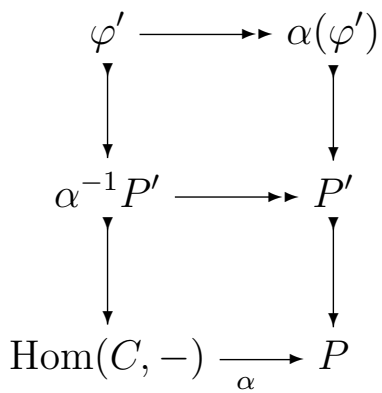

Here $\alpha\left(\varphi^{\prime}\right)$ is the image of the restriction of $\alpha$ to the positive existential formula $\varphi^{\prime} \in J_{\mathbf{D}}(C)$. Since $\alpha\left(\varphi^{\prime}\right)$ is the quotient of a f.p. functor, it must be f.g. But since $P$ is f.p. and our functor category is locally coherent, $\alpha\left(\varphi^{\prime}\right)$ is in fact f.p. Moreover, it is easy to see that it is dense in $P$.

So we can assume that $\mathcal{D}(P)$ is the filter generated by $\left\{\varphi_{\mu} / \tilde{\theta}\right\}_{\mu}$ where $\varphi_{\mu} / \tilde{\theta}$ is dense in $\varphi / \tilde{\theta}$. Then

$$
\operatorname{Nat}(a P, a Q) \cong \underset{\mu}{\lim } \underset{\lambda}{\lim } \operatorname{Nat}\left(\frac{\varphi_{\mu}}{\tilde{\theta}}, \frac{\psi}{\tilde{\eta}_{\lambda}}\right)
$$


We know that any map $\varphi_{\mu} / \tilde{\theta} \rightarrow \psi / \tilde{\eta}_{\lambda}$ is given by a positive existential formula $\rho$ (which defines a map on the whole of $\mathbf{C}$ ). Restricted to $\mathbf{D}, \rho$ will define a map $\varphi / \tilde{\theta} \rightarrow \psi / \tilde{\eta}$. Moreover, any other representative for the class of $\rho$ in the directed colimit will define the same map $\varphi / \tilde{\theta} \rightarrow \psi / \tilde{\eta}$ on $\mathbf{D}$.

Conversely, suppose that $\rho: \varphi / \tilde{\theta} \rightarrow \psi / \tilde{\eta}$ defines a map on D. Without loss, we can asssume that $\forall \boldsymbol{x}, \boldsymbol{y}(\rho(\boldsymbol{x}, \boldsymbol{y}) \rightarrow \psi(\boldsymbol{y}))$ holds on C. Let $\varphi_{0}(\boldsymbol{x})$ be the positive existential formula

$$
\varphi(\boldsymbol{x}) \wedge \exists \boldsymbol{y} \rho(\boldsymbol{x}, \boldsymbol{y})
$$

Then clearly $\varphi_{0} / \tilde{\theta}$ is dense in $\varphi / \tilde{\theta}$. Now let $\eta_{0}(\boldsymbol{y}, \boldsymbol{z})$ be the formula

$$
\exists \boldsymbol{x}, \boldsymbol{x}^{\prime}\left(\theta\left(\boldsymbol{x}, \boldsymbol{x}^{\prime}\right) \wedge \rho(\boldsymbol{x}, \boldsymbol{y}) \wedge \rho\left(\boldsymbol{x}^{\prime}, \boldsymbol{z}\right)\right) \vee \eta(\boldsymbol{y}, \boldsymbol{z})
$$

It is clear that $\tilde{\eta}_{0}(D)=\tilde{\eta}(D)$ for all $D \in \mathbf{D}$ so that $\eta \subseteq \eta_{0} \subseteq E$. Note that $\rho$ defines a map

$$
\varphi_{0} / \tilde{\theta} \rightarrow \psi / \tilde{\eta}_{0}
$$

Thus we obtain a "relativized/localized" version of the results in Part I.

Theorem 3.18. Let $\mathbf{D} \subseteq \mathbf{C}$ be a definable subcategory. The category $\mathrm{fp}\left(\operatorname{Sh}\left(\mathrm{fp} \mathbf{C}, J_{\mathbf{D}}\right)\right)$ has the following logical characterisation. Its objects are pairs of positive existential formulas of the form $\varphi / \tilde{\theta}$ and its morphisms are positive existential formulas which define maps $\varphi / \tilde{\theta} \rightarrow \psi / \tilde{\eta}$ when restricted to $\mathbf{D}$.

\subsection{Finitely generated and coherent sheaves}

The above argument which yielded the characterisation result for f.p. sheaves may also be applied to f.g. sheaves. In fact the argument is slightly simpler. If $P \cong \varphi / E_{1}$ and $Q \cong \psi / E_{2}$ are two f.g. presheaves, then

$$
\operatorname{Nat}(a P, a Q) \cong \underset{\lambda}{\lim } \operatorname{Nat}\left(\varphi_{\lambda} / E_{1}, \psi / E\right)
$$

where $\varphi_{\lambda}$ is dense in $\varphi$, and $E \supseteq E_{2}$ is the closure of $E_{2}$ in $\psi \times \psi($ so $\psi / E \cong$ $Q_{\text {sep }}$ ). Any map $\varphi_{\lambda} / E_{1} \rightarrow \psi / E$ is given by a pe-formula (Proposition 2.9) which, when restricted to $\mathbf{D}$, defines a map $\varphi / E_{1} \rightarrow \psi / E_{2}$. Two formulas representing the same maps in the directed colimit will define the same map on D. 
Conversely if $\rho$ is a pe-formula which defines a map $\varphi / E_{1} \rightarrow \psi / E_{2}$ when restricted to $\mathbf{D}$, then the formula $\varphi_{0}$ expressing (as above) that this map is total is dense in $\varphi$, and the equivalence relation generated by

$$
E_{2}(\boldsymbol{y}, \boldsymbol{z}) \vee\left(\exists \boldsymbol{x}, \boldsymbol{x}^{\prime}\left(E_{1}\left(\boldsymbol{x}, \boldsymbol{x}^{\prime}\right) \wedge \rho(\boldsymbol{x}, \boldsymbol{y}) \wedge \rho\left(\boldsymbol{x}^{\prime}, \boldsymbol{z}\right)\right)\right)
$$

is contained in $E$ (since $E$ is the closure of $E_{2}$ ), so $\rho$ defines a natural transformation $\varphi_{0} / E_{1} \rightarrow \psi / E$. This gives us the following result.

Proposition 3.19. Let $\mathbf{D} \subseteq \mathbf{C}$ be a definable subcategory. The category of finitely generated sheaves $\operatorname{fg}\left(\operatorname{Sh}\left(\mathrm{fp}_{\mathbf{C}} \mathbf{C}, J_{\mathbf{D}}\right)\right)$ is the category with objects $\varphi / E$ where $\varphi$ is a pe-formula and $E$ is a functorial equivalence relation on $\varphi$, and with maps pe-formulas $\rho: \varphi / E_{1} \rightarrow \psi / E_{2}$ which define actual maps on the subcategory $\mathbf{D}$.

With our characterisation of the category of finitely generated sheaves $\mathrm{fg}\left(\operatorname{Sh}\left(\mathrm{fp} \mathbf{C}, J_{\mathbf{D}}\right)\right)$ we can deduce a logical characterisation of the category of coherent sheaves $\operatorname{coh}\left(\operatorname{Sh}\left(\right.\right.$ fp $\left.\left.\mathbf{C}, J_{\mathbf{D}}\right)\right)$.

Proposition 3.20. The coherent objects of $\operatorname{Sh}\left(\mathrm{fp} \mathbf{C}, J_{\mathbf{D}}\right)$ are those isomorphic to one of the form $\varphi / \theta$ where $\varphi, \theta$ are pe and $\theta$ defines an equivalence relation on $\varphi$ when restricted to objects of the definable subcategory $\mathbf{D}$.

Proof. Let $F$ be a coherent sheaf. Then $F$ is f.g. so we can write $F$ in the form $\varphi / E$. Since $F$ is coherent, there is a pullback diagram of the form

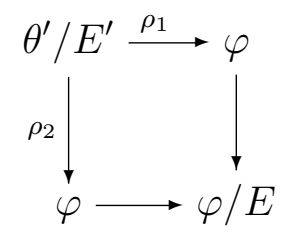

in the sheaf category. By the previous proposition, we can assume that the maps $\rho_{i}$ are pe-formulas which define functions on $\mathbf{D}$. So

$$
E(\boldsymbol{x}, \boldsymbol{y}) \Leftrightarrow \exists \boldsymbol{z}\left(\theta^{\prime}(\boldsymbol{z}) \wedge \rho_{1}(\boldsymbol{z}, \boldsymbol{x}) \wedge \rho_{2}(\boldsymbol{z}, \boldsymbol{y})\right)
$$

holds on D. Let $\theta(\boldsymbol{x}, \boldsymbol{y})$ be the pe-formula on the right hand side. Then $\theta$ defines an equivalence relation on $\mathbf{D}$ and $\varphi / \tilde{\theta} \cong \varphi / E$ in the sheaf category. We can write $\varphi / \tilde{\theta}$ simply as $\varphi / \theta$ where $\theta$ defines an equivalence relation on D. 
Now suppose $F \cong \varphi / \theta$ where $\theta$ defines an equivalence relation on $\mathbf{D}$. We want to show that $F$ is coherent. Let $G \cong \psi / E$ be a f.g. sheaf and let $\rho: \psi / E \rightarrow \varphi / \theta$ be a pe-formula which defines a map on $\mathbf{D}$. If $\gamma(\boldsymbol{x}, \boldsymbol{y})$ is the formula

$$
\exists \boldsymbol{w}, \boldsymbol{z}(\psi(\boldsymbol{x}) \wedge \psi(\boldsymbol{y}) \wedge \rho(\boldsymbol{x}, \boldsymbol{w}) \wedge \rho(\boldsymbol{y}, \boldsymbol{z}) \wedge \theta(\boldsymbol{w}, \boldsymbol{z}))
$$

then $G \times{ }_{F} G$ is a quotient of $\gamma$ in the sheaf category, so $G \times{ }_{F} G$ is finitely generated. Therefore $F$ is coherent as required.

We describe the category $\operatorname{coh}\left(\operatorname{Sh}\left(\mathrm{fp} \mathbf{C}, J_{\mathbf{D}}\right)\right)$ in the following theorem.

Theorem 3.21. Let $\mathbf{D} \subseteq \mathbf{C}$ be definable. The category $\operatorname{coh}\left(\operatorname{Sh}\left(\mathrm{fp} \mathbf{C}, J_{\mathbf{D}}\right)\right)$ has as objects pairs of pe-formulas $\varphi / \theta$ where $\theta$ defines an equivalence relation on $\varphi$ on all objects of $\mathbf{D}$. The maps $\varphi / \theta \rightarrow \psi / \eta$ are given by pe-formulas $\rho$ which define actual functions $\varphi / \theta \rightarrow \psi / \eta$ when restricted to objects of $\mathbf{D}$.

This result can be found in [19] (see p. 269), although it is not so easy to recognise it as the same result. Makkai and Reyes' proof is very different from ours, as is their terminology.

Definable subcategories $\mathbf{D} \subseteq \mathbf{C}$ are categories of models of coherent theories. Given a coherent theory $T$, the category of sheaves $\operatorname{Sh}\left(\mathrm{fp} \mathbf{C}, J_{\operatorname{Mod} T}\right)$ is the classifying topos of $T$. We have shown that the full subcategory of coherent objects in this topos is equivalent to the category with objects

$$
\{\varphi / \theta: \varphi, \theta \text { pe-formulas s.t. } T \vdash(\theta \text { is an equivalence relation on } \varphi)\}
$$

and with $\operatorname{Hom}-\operatorname{sets} \operatorname{Hom}(\varphi / \theta, \psi / \eta)$ of the form

$$
\{\rho: \rho \text { is a pe-formula s.t. } T \vdash(\rho \text { defines a function } \varphi / \theta \rightarrow \psi / \eta)\}
$$

We will denote this category by $T^{\mathrm{eq}+}$ or $\mathbf{D}^{\mathrm{eq}+}$ where $\mathbf{D}=\operatorname{Mod} T$.

\section{Interpretations}

\subsection{Interpretations as functors}

Fix two first-order signatures $\mathcal{L}$ and $\mathcal{K}$. For simplicity of notation we write as if $\mathcal{L}$ and $\mathcal{K}$ are one-sorted but all our definitions and results are easily generalised to many-sorted languages. Let $\mathbf{C}$ be the category of $\mathcal{L}$-structures and $\mathbf{A}$ the category of $\mathcal{K}$-structures. Let $\mathbf{D} \subseteq \mathbf{C}$ and $\mathbf{B} \subseteq \mathbf{A}$ be definable 
subcategories. We define an interpretation of $\mathbf{B}$ in $\mathbf{D}$ to be an exact (i.e. commuting with finite limits and finite colimits) functor

$$
\Gamma: \mathbf{B}^{\mathrm{eq}+} \longrightarrow \mathbf{D}^{\mathrm{eq}+}
$$

Note that although the eq + categories are closed under finite limits, they need not be closed under finite colimits. However it still makes sense to talk of an exact functor between such categories, meaning one which preserves finite limits and such finite colimits as do exist. In practice specific interpretations are usually defined by specifying data as in the next result.

Proposition 4.1. Fix an object $\varphi / \theta$ of $\mathbf{D}^{\mathrm{eq}+}$. Associate to each $n$-ary function symbol $f \in \mathcal{K}$ a map

$$
\Gamma_{f}:\left(\frac{\varphi}{\theta}\right)^{n} \longrightarrow \frac{\varphi}{\theta}
$$

of $\mathbf{D}^{\mathrm{eq}+}$, and to each $m$-ary relation symbol $R \in \mathcal{K}$ an object

$$
\Gamma_{R} \subseteq\left(\frac{\varphi}{\theta}\right)^{m}
$$

such that for each $D \in \mathbf{D}$ the $\mathcal{K}$-structure $\left(\frac{\varphi}{\theta}(D), \Gamma_{f}(D), \Gamma_{R}(D)\right)_{f, R \in \mathcal{K}}$ is in B. Then the function

$$
\begin{aligned}
x=x & \longmapsto \varphi / \theta \\
f & \longmapsto \Gamma_{f} \\
R & \longmapsto \Gamma_{R}
\end{aligned}
$$

has a unique extension to an interpretation $\Gamma: \mathbf{B}^{\mathrm{eq}+} \rightarrow \mathbf{D}^{\mathrm{eq}+}$.

(Constants can be treated as special cases of the above.)

Proof. We extend the function as follows. First put $\Gamma_{(x=x)^{n}}=(\varphi / \theta)^{n}$ for each $n$. Now, any term $t\left(x_{1}, \ldots, x_{n}\right)$ of $\mathcal{K}$ defines a map

$$
t:(x=x)^{n} \longrightarrow x=x
$$

of $\mathbf{B}^{\text {eq+}}$. Suppose that $t$ is the term $f\left(f_{1}(\boldsymbol{x}), \ldots, f_{m}(\boldsymbol{x})\right)$ where $f, f_{1}, \ldots, f_{m}$ are function symbols of $\mathcal{K}$. Then we put

$$
\Gamma_{t}=\Gamma_{f} \circ\left(\Gamma_{f_{1}}, \ldots, \Gamma_{f_{m}}\right)
$$


We now define the action of $\Gamma$ on a term equation $t(\boldsymbol{x})=s(\boldsymbol{x})$ where $\boldsymbol{x}=\left(x_{1}, \ldots, x_{n}\right)$. The functor $t=s$ is an equaliser as shown.

$$
t=s \longrightarrow(x=x)^{n} \stackrel{t}{\underset{s}{\longrightarrow}} x=x
$$

So we define $\Gamma_{t=s}$ to be the equaliser of $\Gamma_{t}$ and $\Gamma_{s}$. Note that $\Gamma_{t=s}$ will indeed be an object of $\mathbf{D}^{\text {eq+ }}$ since this category has all finite limits.

We now consider an atomic formula $R\left(t_{1}, \ldots, t_{k}\right)$ in $m$ free variables. Define $\Gamma_{R\left(t_{1}, \ldots, t_{k}\right)}$ to be $\Gamma_{\left(t_{1}, \ldots, t_{k}\right)}^{-1}\left(\Gamma_{R}\right)$ as in the following diagram.

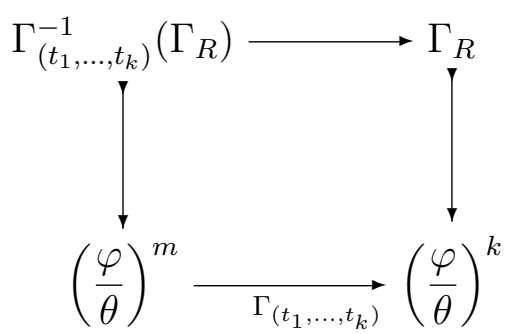

Again, $\Gamma_{R\left(t_{1}, \ldots, t_{k}\right)}$ will be an object of $\mathbf{D}^{\mathrm{eq}+}$.

For a functor $\psi$ of the form $\bigvee_{i=1}^{n} \bigwedge_{j=1}^{m} \psi_{i j}$ where $\psi_{i j}$ is atomic, we define

$$
\Gamma_{\psi}=\bigcup_{i} \bigcap_{j} \Gamma_{\psi_{i j}}
$$

Suppose now that $\psi=\psi(\boldsymbol{x}, \boldsymbol{y})$ where $\boldsymbol{x}$ is of length $n$ and $\boldsymbol{y}$ of length $m$. Then $\Gamma_{\psi}$ embeds in $(\varphi / \theta)^{n+m}$. We define $\Gamma_{\exists \boldsymbol{y} \psi}$ to be the image of $\Gamma_{\psi}$ under the projection map

$$
(\varphi / \theta)^{n+m} \longrightarrow(\varphi / \theta)^{n}
$$

So far, we have extended $\Gamma$ to all positive existential formulas (as well as maps defined by terms). This map corresponds exactly to the syntactic interpretation map of the Reduction Theorem as described in [7, p. 214].

To complete the definition of $\Gamma$, we need to define its action on pairs of positive existential formulas $\psi / \eta$ and maps between them. Note that $\psi / \eta$ is the coequaliser of the two projection maps

$$
\eta \Longrightarrow \psi
$$

so we can define $\Gamma_{\psi / \eta}$ to be the coequaliser of the corresponding projection maps

$$
\Gamma_{\eta} \Longrightarrow \Gamma_{\psi}
$$


The fact that $\eta$ is an equivalence relation on $\psi$ is expressible by a $\mathcal{K}$-formula. Since the above process takes structures in $\mathbf{D}$ to structures in $\mathbf{B}$, the Reduction Theorem gives us that the corresponding $\mathcal{L}$-formula holds on $\mathbf{D}$ so that $\Gamma_{\eta}$ is an equivalence relation on $\Gamma_{\psi}$. Hence $\Gamma_{\psi / \eta} \cong \Gamma_{\psi} / \Gamma_{\eta}$.

So how should $\Gamma$ act on a positive existential map

$$
\rho: \psi / \eta \rightarrow \chi / \zeta
$$

of $\mathbf{B}^{\text {eq+}}$ ? Note that $\rho$ defines a subfunctor of $\psi \times \chi$ which satisfies the $\mathcal{K}$ formulas

$$
\begin{gathered}
\forall \boldsymbol{x}(\psi(\boldsymbol{x}) \rightarrow \exists \boldsymbol{y} \rho(\boldsymbol{x}, \boldsymbol{y})) \\
\forall \boldsymbol{x}, \boldsymbol{x}^{\prime}, \boldsymbol{y}, \boldsymbol{y}^{\prime}\left(\eta\left(\boldsymbol{x}, \boldsymbol{x}^{\prime}\right) \wedge \rho(\boldsymbol{x}, \boldsymbol{y}) \wedge \rho\left(\boldsymbol{x}^{\prime}, \boldsymbol{y}^{\prime}\right) \rightarrow \zeta\left(\boldsymbol{y}, \boldsymbol{y}^{\prime}\right)\right)
\end{gathered}
$$

on the definable subcategory $\mathbf{B}$. Regarding $\rho$ as an object of $\mathbf{B}^{\mathrm{eq}+}, \Gamma_{\rho}$ defines a subfunctor of $\Gamma_{\psi} \times \Gamma_{\chi}$. The two $\mathcal{K}$-formulas above have obvious translations to $\mathcal{L}$-formulas which say exactly that $\Gamma_{\rho}$ defines a function

$$
\Gamma_{\rho}: \Gamma_{\psi / \eta} \longrightarrow \Gamma_{\chi / \zeta}
$$

These $\mathcal{L}$-formulas will hold on $\mathbf{D}$ by the Reduction Theorem. It is easy to check that this action of $\Gamma$ on maps $\rho$ is functorial.

This extension to a functor $\Gamma: \mathbf{B}^{\mathrm{eq}+} \rightarrow \mathbf{D}^{\mathrm{eq}+}$ is clearly the unique such extension to a functor commuting with finite limits and finite colimits.

The above proof shows, amongst other things, that the interpretation functor $\Gamma$ takes positive existential formulas to their syntactic translations in the sense of the Reduction Theorem (see [7]). We will henceforth refer to the following restricted form of this theorem as the Reduction Theorem. Its proof is a direct consequence of the above proof and the proof in [7]. The notation $\Gamma_{\varphi}$ is as in the proof of Proposition 4.1 .

Theorem 4.2 (Reduction Theorem). Let $\Gamma: \mathbf{B}^{\mathrm{eq}+} \rightarrow \mathbf{D}^{\mathrm{eq}+}$ be an exact functor. Fix $D \in \mathbf{D}$ and let $B=\left(\Gamma_{x=x}(D), \Gamma_{f}(D), \Gamma_{R}(D)\right)_{f, R \in \mathcal{K} \text {. Then, for }}$ any $\left(\left[\boldsymbol{a}_{1}\right], \ldots,\left[\boldsymbol{a}_{n}\right]\right) \in \Gamma_{x=x}(D)$, and any pe $\mathcal{K}$-formula $\varphi\left(x_{1}, \ldots, x_{n}\right)$ we have

$$
B \models \varphi\left(\left[\boldsymbol{a}_{1}\right], \ldots,\left[\boldsymbol{a}_{n}\right]\right) \Leftrightarrow D \models \Gamma_{\varphi}\left(\boldsymbol{a}_{1}, \ldots, \boldsymbol{a}_{n}\right)
$$

¿From this it follows that an exact functor $\Gamma: \mathbf{B}^{\mathrm{eq}+} \rightarrow \mathbf{D}^{\mathrm{eq}+}$ induces a functor $\mathbf{D} \rightarrow \mathbf{B}$ which does indeed correspond to an interpretation in the sense of model theory: we are interpreting $\mathbf{B}$ in $\mathbf{D}$ in a definable way. 
Proposition 4.3. Let $\mathbf{B}$ and $\mathbf{D}$ be definable subcategories as above. An exact functor $\Gamma: \mathbf{B}^{\mathrm{eq}+} \rightarrow \mathbf{D}^{\mathrm{eq}+}$ induces a functor $\Gamma_{x=x}: \mathbf{D} \rightarrow \mathbf{B}$ via

$$
D \longmapsto\left(\Gamma_{x=x}(D), \Gamma_{f}(D), \Gamma_{R}(D)\right)_{f, R \in \mathcal{K}}
$$

Moreover, this functor commutes with directed colimits and pure embeddings.

Proof. We first need to show that for every $D \in \mathbf{D}$ the $\mathcal{K}$-structure $\left(\Gamma_{x=x}(D), \Gamma_{f}(D), \Gamma_{R}(D)\right)_{f, R \in \mathcal{K}}$ is in B. Suppose $\mathbf{B}=\operatorname{Mod} T$ for a coherent theory $T$. Choose a coherent axiom $\varphi \rightarrow \psi$ of $T$. Let $\rho(\boldsymbol{x}, \boldsymbol{y})$ be the relation defined by the pe-formula

$$
\varphi(\boldsymbol{x}) \wedge \psi(\boldsymbol{y}) \wedge \boldsymbol{x}=\boldsymbol{y}
$$

Then $\rho$ defines a monomorphism $\varphi \rightarrow \psi$ in $\mathbf{B}^{\text {eq }+}$. By left exactness of $\Gamma, \rho$ is taken to a monomorphism $\Gamma_{\rho}: \Gamma_{\varphi} \rightarrow \Gamma_{\psi}$ in $\mathbf{D}^{\text {eq }+}$, so

$$
\mathrm{D} \models \Gamma_{\varphi} \rightarrow \Gamma_{\psi}
$$

By the reduction theorem,

$$
\left(\Gamma_{x=x}(D), \Gamma_{f}(D), \Gamma_{R}(D)\right)_{f, R \in \mathcal{K}} \models \varphi \rightarrow \psi
$$

for every $D \in \mathbf{D}$.

That the association

$$
D \longmapsto\left(\Gamma_{x=x}(D), \Gamma_{f}(D), \Gamma_{R}(D)\right)_{f \in \mathcal{K}}
$$

is functorial is obvious. So we indeed have a functor $\Gamma_{x=x}: \mathbf{D} \rightarrow \mathbf{B}$. It remains to show that this functor commutes with directed colimits and pure embeddings.

Since $\Gamma_{x=x}, \Gamma_{f}$ and $\Gamma_{R}$ have expressions as quotients of pe-formulas, which we know commute with directed colimits, we have the first statement. As for the second, suppose that $D \subseteq D^{\prime}$ is a pure embedding in $\mathbf{D}$. Then $\Gamma_{x=x}(D) \rightarrow \Gamma_{x=x}\left(D^{\prime}\right)$ is a monomorphism. For suppose $\Gamma_{x=x} \cong \varphi / \theta$. Let $\boldsymbol{a}, \boldsymbol{b} \in \varphi(D)$ such that $D \models \theta(\boldsymbol{a}, \boldsymbol{b})$. By purity, $D \models \theta(\boldsymbol{a}, \boldsymbol{b})$, so $\varphi / \theta(D) \rightarrow$ $\varphi / \theta\left(D^{\prime}\right)$ is monic.

To show that the embedding $\varphi / \theta(D) \rightarrow \varphi / \theta\left(D^{\prime}\right)$ is pure, let $\psi$ be a positive existential $\mathcal{K}$-formula and let $\boldsymbol{a}_{1}, \ldots, \boldsymbol{a}_{n} \in \varphi(X)$ such that

$$
\left(\frac{\varphi}{\theta}\left(D^{\prime}\right), \Gamma_{f}\left(D^{\prime}\right), \Gamma_{R}\left(D^{\prime}\right)\right)_{f, R \in \mathcal{K}} \models \psi\left(\left[\boldsymbol{a}_{1}\right], \ldots,\left[\boldsymbol{a}_{n}\right]\right)
$$


We can now apply the reduction theorem to get

$$
D^{\prime} \models \Gamma_{\psi}\left(\boldsymbol{a}_{1}, \ldots, \boldsymbol{a}_{n}\right)
$$

Since $D \subseteq D^{\prime}$ is pure, we have

$$
D \models \Gamma_{\psi}\left(\boldsymbol{a}_{1}, \ldots, \boldsymbol{a}_{n}\right)
$$

and so, by applying the reduction theorem once more, we have that

$$
\left(\frac{\varphi}{\theta}(D), \Gamma_{f}(D), \Gamma_{R}(D)\right)_{f, R \in \mathcal{K}} \models \psi\left(\left[\boldsymbol{a}_{1}\right], \ldots,\left[\boldsymbol{a}_{n}\right]\right)
$$

as required.

It is easy to see that interpretations behave well with respect to composition. For suppose that we have interpretations

$$
\mathrm{B}^{\mathrm{eq}+} \stackrel{\Gamma}{\longrightarrow} \mathrm{D}^{\mathrm{eq}+} \stackrel{\Delta}{\longrightarrow} \mathbf{F}^{\mathrm{eq}+}
$$

where $\Gamma_{x=x}=\varphi / \theta$ and $\Delta_{x=x}=\psi / \eta$. Then one can check that $\Gamma_{x=x} \circ \Delta_{x=x}=$ $\Delta_{\varphi / \theta}$ so

$$
(\Delta \circ \Gamma)_{x=x}=\Gamma_{x=x} \circ \Delta_{x=x}
$$

So now that we have a nice definition of interpretation, we can give a relatively simple and natural definition of a bi-interpretation. We say that a pair $(\Gamma, \Delta)$ is a bi-interpretation between definable subcategories $\mathbf{B}$ and $\mathbf{D}$ if

$$
\mathbf{B}^{\mathrm{eq}+} \underset{\Delta}{\stackrel{\Gamma}{\rightleftarrows}} \mathrm{D}^{\mathrm{eq}+}
$$

are interpretations such that

$$
\begin{aligned}
& (\Gamma \circ \Delta)_{x=x} \cong x=x \\
& (\Delta \circ \Gamma)_{x=x} \cong x=x
\end{aligned}
$$

as objects of $\mathbf{D}^{\mathrm{eq}+}$ and $\mathbf{B}^{\mathrm{eq}+}$ respectively. So these natural isomorphisms will be given by positive existential formulas, corresponding to the notion of definable isomorphism in the classical definition of a bi-interpretation.

We shall give some examples of interpretations and bi-interpretations in the next section. But first we show that one can recover a definable subcategory $\mathbf{D}$ as the category of exact functors on $\mathbf{D}^{\mathrm{eq}+}$. Our notation for this category of exact functors is $\operatorname{Ex}\left(\mathbf{D}^{\mathrm{eq}+}\right.$, Set $)$. As mentioned earlier, in the additive case this was noticed by Herzog but in the category-theoretic literature such results go back at least as far as [19]. Indeed, the next result is the finitary case of $[8,5.10($ ii) $]$. 
Proposition 4.4. Given a definable subcategory $\mathbf{D}$ in a first-order language $\mathcal{L}$,

$$
\mathbf{D} \simeq \operatorname{Ex}\left(\mathbf{D}^{\mathrm{eq}+}, \mathbf{S e t}\right)
$$

Proof. We define a functor

$$
\mathrm{ev}: \mathbf{D} \longrightarrow\left(\mathbf{D}^{\mathrm{eq}+}, \mathbf{S e t}\right)
$$

by $\mathrm{ev}_{D}(F)=F(D)$ and $\mathrm{ev}_{f}(F)=F(f)$. It is easily checked that $\mathrm{ev}_{D}$ is an exact functor $\mathbf{D}^{\mathrm{eq}+} \rightarrow$ Set, so ev maps to $\operatorname{Ex}\left(\mathbf{D}^{\mathrm{eq}+}\right.$, Set $)$. It is also easy to see that ev is a faithful functor. For suppose $f, g: X \rightarrow Y$ are parallel arrows in $\mathbf{D}$ such that $\mathrm{ev}_{f}=\mathrm{ev}_{g}$. Then $\mathrm{ev}_{f}(x=x)=\mathrm{ev}_{g}(x=x)$, so $f=g$.

Now suppose that we have a map $\Phi: \mathrm{ev}_{D} \rightarrow \mathrm{ev}_{E}$ in $\left(\mathbf{D}^{\mathrm{eq}+}\right.$, Set). Evaluate $\Phi$ at $(x=x) \in \mathbf{D}^{\text {eq+ }}$ to obtain a map $f: D \rightarrow E$ of sets. For each $n$ consider the map $\bigwedge_{i=1}^{n}\left(x_{i}=x_{i}\right) \rightarrow\left(x_{k}=x_{k}\right)$ of $\mathbf{D}^{\text {eq+ }}$ corresponding to projection onto the $k$ th factor. The naturality of $\Phi$ gives us the following commutative square.

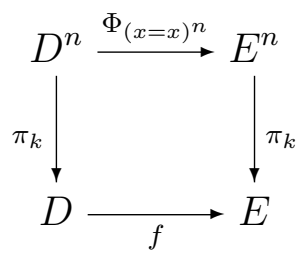

By letting $k$ vary we see that $\Phi_{(x=x)^{n}}=f^{n}$.

Now, for every $n$-ary function symbol $\sigma:(x=x)^{n} \rightarrow(x=x)$ naturality of $\Phi$ gives a commutative square

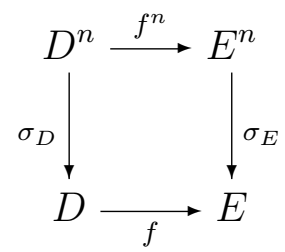

and, for every relation symbol $R \subseteq(x=x)^{m}$, a commutative square

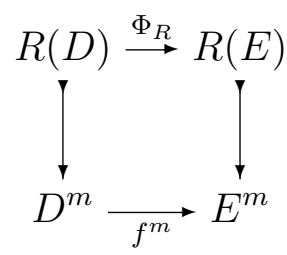


Thus $f$ is a homomorphism $D \rightarrow E$. That is, $f \in \mathbf{D}$.

Since every pe-formula $\varphi$ in $n$ variables defines a subobject of $(x=x)^{n}$ in $\mathbf{D}^{\text {eq+ }}$, it is easy to see that $\Phi_{\varphi}$ is the restriction of $f^{n}$ to $\varphi(X)$. Also since $\varphi / \theta$ is a quotient object of $\varphi$ in $\mathbf{D}^{\mathrm{eq}+}$, one can deduce that $\Phi_{\varphi / \theta}$ takes an equivalence class $[\boldsymbol{x}]$ in $\varphi / \theta(X)$ to the equivalence class $\left[f^{n}(\boldsymbol{x})\right]$ in $\varphi / \theta(Y)$. Hence $\Phi=\mathrm{ev}_{f}$. That is, the functor ev is full.

We now show that ev is an equivalence. Let $\Gamma: \mathbf{D}^{\text {eq }+} \rightarrow$ Set be any exact functor. Let $D$ be the $\mathcal{L}$-structure $(\Gamma(x=x), \Gamma(f), \Gamma(R))_{f, R \in \mathcal{L}}$. Using the exactness of $\Gamma$, it is easily checked that $\Gamma(\varphi)=\varphi(D)$ for every pe-formula $\varphi$. We claim that $D \in \mathbf{D}$. Suppose that $\mathbf{D}=\operatorname{Mod} T$ for a coherent theory $T$. Let $\varphi \rightarrow \psi$ be a coherent axiom of $T$. Let $\rho(\boldsymbol{x}, \boldsymbol{y})$ be the relation defined by

$$
\varphi(\boldsymbol{x}) \wedge \psi(\boldsymbol{y}) \wedge \boldsymbol{x}=\boldsymbol{y}
$$

Then $\rho$ defines a monomorphism $\varphi \rightarrow \psi$ in $\mathbf{D}^{\mathrm{eq}+}$. By exactness of $\Gamma, \Gamma(\rho)$ : $\Gamma(\varphi) \rightarrow \Gamma(\psi)$ is a monomorphism of sets, so $\Gamma(\varphi) \subseteq \Gamma(\psi)$, i.e. $\varphi(D) \subseteq \psi(D)$, that is

$$
D \models \varphi \rightarrow \psi
$$

Thus $D \in \mathbf{D}=\operatorname{Mod} T$. Clearly $\Gamma \cong \mathrm{ev}_{D}$, so ev is indeed an equivalence as required.

\subsection{Examples of interpretations}

Let $\mathcal{L}=\mathcal{K}$ be the language of rings. Let $\mathbf{D}$ be the category of real closed fields and $\mathbf{B}$ the category of algebraically closed fields. It is easy to see that $\mathbf{D}$ and $\mathbf{B}$ are definable subcategories. The standard two dimensional interpretation of an algebraically closed field in a real closed field is an instance of an interpretation in our sense.

In fact any classical interpretation by positive existential formulas of a $\mathcal{K}$-structure $B$ in an $\mathcal{L}$-structure $D$ can be thought of as an interpretation in our sense between the category of $\mathcal{K}$-structures and a suitable definable subcategory $\mathbf{D}$ of the category of $\mathcal{L}$-structures. For suppose that

$$
B \cong\left(\frac{\varphi}{\theta}(D), \Gamma_{f}(D), \Gamma_{R}(D)\right)_{f, R \in \mathcal{K}}
$$

where $\theta(D)$ is an equivalence relation on $\varphi(D)$. Let $\mathbf{D}$ be the category of $\mathcal{L}$-structures on which $\theta$ is an equivalence relation and the $\Gamma_{f}$ are definable maps $(\varphi / \theta)^{n} \rightarrow \varphi / \theta$, where $n$ is the arity of $f$. The sentences expressing 
these facts can be checked to be coherent sentences. This implies that $\mathbf{D}$ is a definable subcategory and it can easily be seen that this is an instance of an interpretation $(\mathcal{K}-\mathbf{S t r})^{\text {eq }+} \rightarrow \mathbf{D}^{\text {eq }+}$ via the map

$$
\begin{aligned}
x=x & \longmapsto \varphi / \theta \\
(f \in \mathcal{K}) & \longmapsto \Gamma_{f}
\end{aligned}
$$

We now look at an example of a bi-interpretation. This example shows that our theory is also well-suited to what is traditionally regarded as the domain of abelian model/category theory. Let $Q$ be the quiver

$$
1 \bullet \underset{\beta}{\stackrel{\alpha}{\rightleftarrows}} \bullet 2
$$

and let $k$ be any field. Then the path algebra of $Q$ over $k$, written $k Q$, has a basis $\left\{e_{1}, e_{2}, \alpha, \beta\right\}$ over $k$. For any $k Q$-module $M$, left multiplication by $\alpha$ and $\beta$ restricted to $e_{1} M$ define maps of $e_{1} M$ into $e_{2} M$. Let $\mathbf{D}$ be the definable subcategory of $k Q$-Mod consisting of those modules in which this restriction of $\alpha$ is invertible. Define the interpretation

$$
\Gamma:(k[X]-\mathbf{M o d})^{\mathrm{eq}+} \longrightarrow \mathbf{D}^{\mathrm{eq}+}
$$

by putting $\Gamma_{x=x}=\exists z\left(x=e_{2} z\right)$ and $\Gamma_{X}(x, y)=\exists z\left(\alpha e_{1} z=x \wedge \beta e_{1} z=y\right)$. In other words, $\Gamma_{x=x}(M)=e_{2} M$ and $\Gamma_{X}=\beta \alpha^{-1}: e_{2} M \rightarrow e_{2} M$.

Now let $\Delta$ be the interpretation

$$
\Delta: \mathbf{D}^{\mathrm{eq}+} \longrightarrow(k[X]-\mathbf{M o d})^{\mathrm{eq}+}
$$

defined by $\Delta_{x=x}(M)=(x=x \wedge y=y)$ and

$$
\begin{aligned}
& \Delta_{\alpha}:(x, y) \longmapsto(0, x) \\
& \Delta_{\beta}:(x, y) \longmapsto(0, X(x)) \\
& \Delta_{e_{1}}:(x, y) \longmapsto(x, 0) \\
& \Delta_{e_{2}}:(x, y) \longmapsto(0, y)
\end{aligned}
$$

One can check that with this definition one does indeed get a $k Q$-module structure on the direct sum $M \oplus M$ of the $k[X]$-module $M$. We claim that the pair $(\Gamma, \Delta)$ gives a bi-interpretation between the categories $\mathbf{D}$ and $k[X]$-Mod. To see this, note that $\Delta_{x=x} \circ \Gamma_{x=x}(M)=e_{2} M \oplus e_{2} M$ and the natural isomorphism

$$
M \stackrel{\cong}{\longrightarrow} e_{2} M \oplus e_{2} M
$$

is given by $x \mapsto\left(\alpha e_{1} x, e_{2} x\right)$.

Conversely, $\Gamma_{x=x} \circ \Delta_{x=x}(M)=M$. So we do indeed have a bi-interpretation. 


\subsection{Interpretations and coherent morphisms}

Recall that a coherent topos is a category of sheaves for a coherent site. It is a result of topos theory (see [10, Vol. 2, p. 910]) that any coherent topos is the classifying topos of a coherent theory. Hence, any coherent topos will be of the form $\operatorname{Sh}\left(\mathrm{fp} \mathbf{C}, J_{\mathbf{D}}\right)$ for an LFP category $\mathbf{C}$ and a definable subcategory $\mathbf{D} \subseteq \mathbf{C}$, since this category of sheaves is precisely the classifying topos for the coherent theory defining $\mathbf{D}$ (see [19, p. 293]). It follows that the results of this chapter apply to general coherent toposes.

Let $\mathcal{E}$ be a coherent topos and let $J$ be the finite type right Grothendieck topology on $\operatorname{coh} \mathcal{E}$ generated by finite epimorphic families. More precisely, we say that $S \subseteq \operatorname{Hom}(-, C)$ is in $J(C)$ if and only if there are finitely many maps $s_{i} \in S$ such that $\coprod_{i} s_{i}$ is an epimorphism onto $C$. Then, it turns out that $\mathcal{E} \simeq \operatorname{Sh}(\operatorname{coh} \mathcal{E}, J)$ (see [9, 7.3.1], [20, p. 1]). Suppose now that $\mathcal{E}$ and $\mathcal{F}$ are two coherent toposes and $\Gamma: \operatorname{coh} \mathcal{E} \rightarrow \operatorname{coh} \mathcal{F}$ is an interpretation. Then since $\Gamma$ commutes with finite colimits, it will take finite epimorphic families to finite epimorphic families. Let $J$ and $K$ be the finite type right Grothendieck topologies generated by finite epimorphic families on coh $\mathcal{E}$ and coh $\mathcal{F}$ respectively. Then $\Gamma$ has the property that if $S \in J(C)$, then the set

$$
\{\Gamma(s): s \in S\}
$$

generates a cover of $\Gamma(C)$ (i.e. the smallest subfunctor of $\operatorname{Hom}(-, \Gamma(C))$ containing the set is a cover of $\Gamma(C))$.

Whenever there are two small categories with finite limits $\mathbf{A}$ and $\mathbf{B}$, both endowed with a Grothendieck topology, and a left exact functor $F: \mathbf{A} \rightarrow \mathbf{B}$ such that for any $A \in \mathbf{A}$ and cover $S$ of $A$ the set $\{F(s): s \in S\}$ generates a cover of $F(A), F$ is said to be a morphism of sites. So $\Gamma$ above is a morphism of sites $(\operatorname{coh} \mathcal{E}, J) \rightarrow(\operatorname{coh} \mathcal{F}, K)$. As such, it will induce a map of sheaves

$$
\Phi: \operatorname{Sh}(\operatorname{coh} \mathcal{F}, K) \longrightarrow \operatorname{Sh}(\operatorname{coh} \mathcal{E}, J)
$$

The direct image functor $\Phi_{*}$ is simply $\Phi_{*}(F)=F \circ \Gamma$. The inverse image functor $\Phi^{*}$ is slightly more complicated and its definition requires left Kan extensions (see [10, Vol. 1, A4.1.4]). However, in this case $\mathcal{E} \simeq \operatorname{Sh}(\operatorname{coh} \mathcal{E}, J$ ), and $\Phi^{*} \Gamma_{\operatorname{coh} \varepsilon}=\Gamma$. In particular $\Phi^{*}$ takes coherent objects to coherent objects. Any geometric morphism between coherent toposes which has the property that the inverse image functor restricts to a functor on coherent objects is called a coherent morphism. So any interpretation $\Gamma: \operatorname{coh} \mathcal{E} \rightarrow \operatorname{coh} \mathcal{F}$ induces 
a coherent morphism $\mathcal{F} \rightarrow \mathcal{E}$, and conversely, any coherent morphism $\Phi$ : $\mathcal{F} \rightarrow \mathcal{E}$ induces an interpretation $\Phi^{*} \uparrow_{\operatorname{coh} \mathcal{E}} \operatorname{coh} \mathcal{E} \rightarrow \operatorname{coh} \mathcal{F}$ (since the inverse image functor preserves finite limits by definition and preserves colimits by the fact that it is a left adjoint).

In summary, our interpretation functors defined at the start of this section correspond exactly to coherent geometric morphisms between coherent toposes (objects which have been studied in the category-theoretic literature). As we have seen, these abstractly defined maps can be regarded as genuine model-theoretic interpretations by positive existential formulas. The relationship between our interpretations and coherent morphisms was suggested to us by Tibor Beke.

\section{References}

[1] Adámek, J. and Rosický, J., Locally Presentable and Accessible Categories, London Math. Soc. Lecture Notes Ser., Vol. 189, Cambridge University Press, 1994.

[2] Auslander, M., "Large modules over Artin algebras", pp. 1-17 in Algebra, Topology and Category Theory, Academic Press, New York, 1976.

[3] Borceux, F., Handbook of Categorical Algebra, Vols 1-3, Encyclopedia of Mathematics and Applications, Cambridge University Press, 1994.

[4] Feferman, S. and Vaught, R.L., "The first order properties of algebraic systems", Fund. Math., 47 (1959), 57-103.

[5] Burke, K., Some Model-Theoretic Properties of Functor Categories for Modules, Doctoral Thesis, University of Manchester, 1994.

[6] Herzog, I., "Elementary duality of modules", Trans. Amer. Math. Soc., 340 (1993), 37-69.

[7] Hodges, W., Model Theory, Encyclopedia of Mathematics and Applications, Vol. 42, Cambridge University Press, 1993.

[8] Hu, H., "Dualities for accessible categories", pp. 211-242 in Canad. Math. Soc. Conf. Proc., Vol. 13, 1992. 
[9] Johnstone, P. T., Topos Theory, LMS Monographs no. 10, Academic Press, 1977.

[10] Johnstone, P. T., Sketches of an Elephant: A Topos Theory Compendium, Vols. 1 \& 2, Oxford Logic Guides nos. 43 \& 44, Oxford University Press, 2002.

[11] Keisler, H.J., "Theory of models with generalized atomic formulas", J. Symbolic Logic, 25 (1) (1960), 1-26.

[12] Keisler, H.J. and Chang, C.C., Model Theory, third edition, Studies in Logic and Foundations of Mathematics, Vol. 73, Elsevier Science Publishers B.V., 1990.

[13] Krause, H., "Functors on locally finitely presented categories", Colloq. Math., 75 (1998), 105-132.

[14] MacLane, S., Categories for the Working Mathematician, SpringerVerlag, 1971.

[15] MacLane, S. and Moerdijk, I., Sheaves in Geometry and Logic, SpringerVerlag, 1992.

[16] Makkai, M., "Strong conceptual completeness for first-order logic", Ann. Pure Appl. Logic, 40 (1988), 167-215.

[17] Makkai, M., "A theorem on Barr-exact categories with an infinitary generalization", Ann. Pure Appl. Logic, 47 (1990), 225-268.

[18] Makkai, M. and Paré, R., Accessible Categories: the Foundations of Categorical Model Theory, Contemp. Math., Vol. 104, American Mathematical Society, Providence, RI, 1989.

[19] Makkai, M. and Reyes, G., First Order Categorical Logic, Springer Lecture Notes in Mathematics, vol. 611, 1977.

[20] Moerdijk, I. and Vermeulen, J. J. C., "Proof of a Conjecture of Pitts", preprint.

[21] Prest, M., "Definable additive categories: purity and model theory", preprint, University of Manchester, 2006. 
[22] Prest, M., Purity, Spectra and Localisation, book in preparation.

[23] Prest, M. Model Theory and Modules, London Math. Soc. Lecture Notes Ser., Vol. 130, Cambridge University Press, 1988.

[24] Rosický, J., Adámek, J. and Borceux, F., "More on injectivity in locally presentable categories", Th. Appl. Categories, 10 (2002), 148-161.

[25] Rothmaler, Ph., "Purity in model theory", pp. 445-469 in Advances in Algebra and Model Theory, Gordon and Breach, 1997. 